\title{
Functional architecture underlying binocular coordination of eye position and velocity in the larval zebrafish hindbrain
}

\author{
Christian Brysch ${ }^{1,2}$, Claire Leyden ${ }^{1,2}$ and Aristides B. Arrenberg ${ }^{1 *}$ (D)
}

\begin{abstract}
Background: The oculomotor integrator $(\mathrm{OI})$ in the vertebrate hindbrain transforms eye velocity input into persistent position coding output, which plays a crucial role in retinal image stability. For a mechanistic understanding of the integrator function and eye position control, knowledge about the tuning of the Ol and other oculomotor nuclei is needed. Zebrafish are increasingly used to study integrator function and sensorimotor circuits, yet the precise neuronal tuning to motor variables remains uncharacterized.

Results: Here, we recorded cellular calcium signals while evoking monocular and binocular optokinetic eye movements at different slow-phase eye velocities. Our analysis reveals the anatomical distributions of motoneurons and internuclear neurons in the nucleus abducens as well as those of oculomotor neurons in caudally adjacent hindbrain volumes. Each neuron is tuned to eye position and/or velocity to variable extents and is only activated after surpassing particular eye position and velocity thresholds. While the abducens (rhombomeres 5/6) mainly codes for eye position, in rhombomeres $7 / 8$, a velocity-to-position coding gradient exists along the rostro-caudal axis, which likely corresponds to the oculomotor structures storing velocity and position, and is in agreement with a feedforward mechanism of persistent activity generation. Position encoding neurons are recruited at eye position thresholds distributed across the behaviourally relevant dynamic range, while velocity-encoding neurons have more centred firing thresholds for velocity. In the abducens, neurons coding exclusively for one eye intermingle with neurons coding for both eyes. Many of these binocular neurons are preferentially active during conjugate eye movements and less active during monocular eye movements. This differential recruitment during monocular versus conjugate tasks represents a functional diversification in the final common motor pathway.

Conclusions: We localized and functionally characterized the repertoire of oculomotor neurons in the zebrafish hindbrain. Our findings provide evidence for a mixed but task-specific binocular code and suggest that generation of persistent activity is organized along the rostro-caudal axis in the hindbrain.
\end{abstract}

Keywords: Oculomotor system, Hindbrain, Vertebrate, Binocular, Velocity, Neural integrator, Motor, Calcium imaging

\section{Background}

The oculomotor system is responsible for moving the eyes in vertebrates and is highly conserved across species. Zebrafish are increasingly used to improve our understanding of the oculomotor population code and eye movement control [1-6].

The oculomotor system for horizontal eye movements consists of multiple elements (Fig. 1a). It is responsible

\footnotetext{
* Correspondence: aristides.arrenberg@uni-tuebingen.de

${ }^{1}$ Werner Reichardt Centre for Integrative Neuroscience and Institute for

Neurobiology, University of Tübingen, 72076 Tübingen, Germany

Full list of author information is available at the end of the article
}

for generating and maintaining stable eye positions as well as eye movements during saccades, optokinetic and vestibulo-ocular reflexes (OKR, VOR), and other behaviours. The lateral and medial rectus (LR, MR), which represent the extraocular eye muscles responsible for horizontal eye movements, are controlled by motoneurons $(\mathrm{MN})$ in the nucleus abducens $(\mathrm{ABN})$ and the oculomotor nucleus (OMN), respectively. The OMN MNs are activated by internuclear neurons (INN) residing in the contralateral $\mathrm{ABN}$. The $\mathrm{ABN}$ receives direct or indirect input from a range of structures such as the burst (B) system for driving saccades, the horizontal eye

(c) The Author(s). 2019 Open Access This article is distributed under the terms of the Creative Commons Attribution 4.0 International License (http://creativecommons.org/licenses/by/4.0/), which permits unrestricted use, distribution, and 

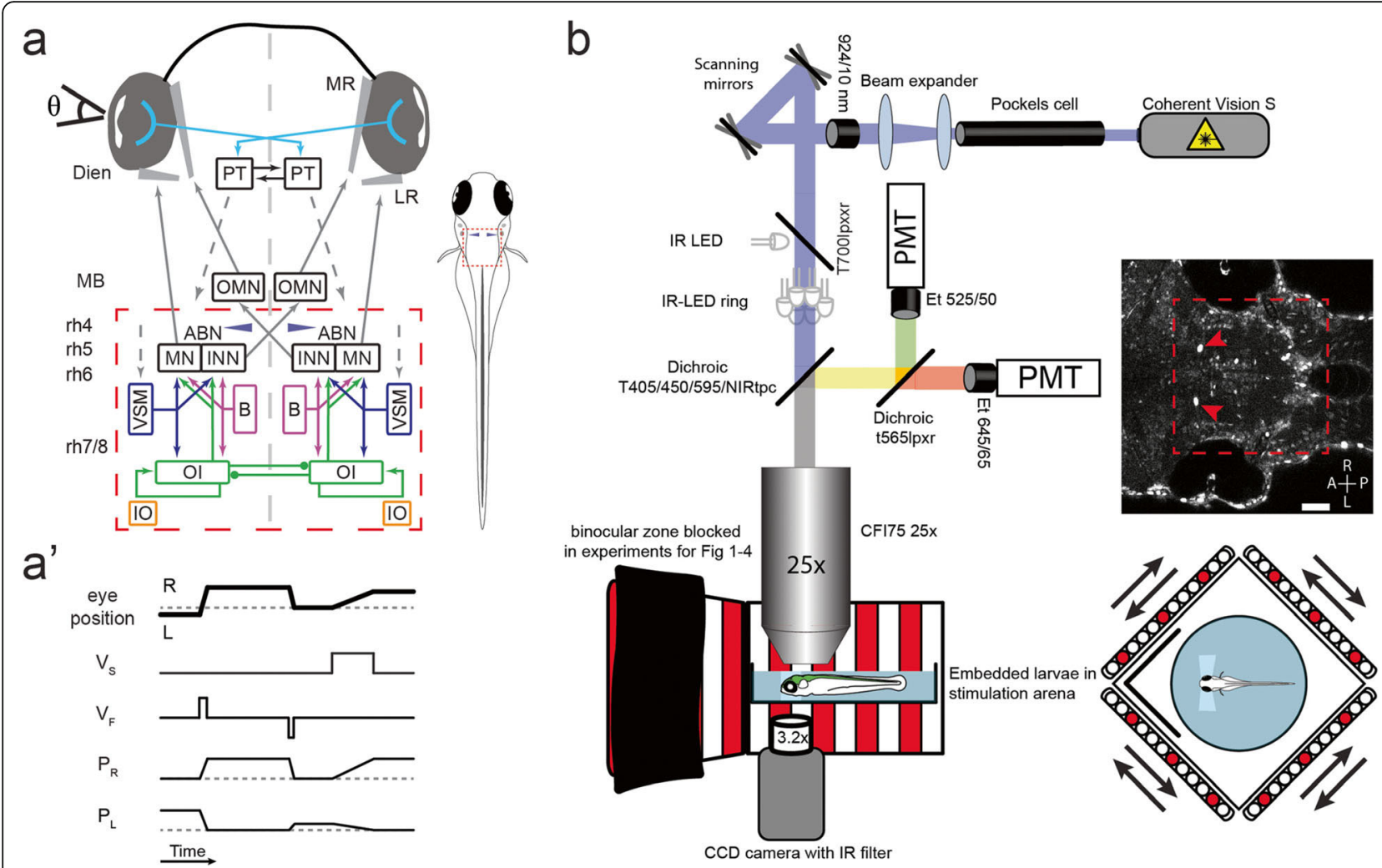

Fig. 1 Setup and circuit overview. a Simplified circuit schematic for horizontal eye movements. Red dashed rectangle represents imaged brain area; blue cones show location of Mauthner cells. ABN, abducens nucleus; B, burst neurons; Dien, diencephalon; INN, internuclear neurons; IO, inferior olive; LR, lateral rectus; MB, midbrain; MN, motoneurons; MR, medial rectus; OMN, nucleus oculomotorius; Ol, oculomotor integrator; PT, pretectum; rh 4-8, rhombomeres 4-8; VSM, velocity storage mechanism; $\Theta$, eye position. Note that the connection from the VSM to the ABN in zebrafish is probably indirect [7]. Dashed arrows indicate direct or indirect inputs from upstream visual brain areas [8, 9]. a' Simplified schematic response profiles for hindbrain oculomotor neurons during eye position changes. Dashed line represents an eye position or velocity of 0 . $L$, left; $P_{L / R}$, Position coding neurons left/right, note that $P_{L}$ and $P_{R}$ have different firing thresholds; $R$, right; $V_{F}$, fast (burst) velocity neurons; $V_{S}$, slow velocity neurons. $\mathbf{b}$ Schematic of microscopy setup. Agarose-embedded zebrafish larvae were visually stimulated, while eye movements were recorded from below, and cellular calcium signals were recorded from above via a two-photon microscope. Setup not drawn to scale, binocular zone excluded for experiment with monocular stimulation only, scale bar $50 \mu \mathrm{m}$, red dashed rectangle represents imaged brain area, red arrows show GCaMP expression in the nuclei of the Mauthner cells, which served as a landmark (blue cones in a and in cell maps). A, anterior; L, left; P, posterior; PMT, photomultiplier tubes; R, right

velocity-to-position neural integrator (termed oculomotor integrator, OI) for maintaining eye positions (P), and the velocity storage mechanism (VSM) associated with slow phase eye velocities (V) during optokinetic and vestibular responses.

The oculomotor integrator is of particular interest, as its persistent firing and dynamic integration of inputs manifest a short-term memory of eye position. It mathematically integrates eye velocity inputs in order to generate a neural representation of eye position via persistent firing $[10,11]$. Its mechanisms of operation [12-14] are not fully understood and could provide insights into memory functions of other, higher, brain areas as well. The OI neurons in zebrafish are functionally heterogeneous, and their differential function is likely related to the mechanism of integration. The zebrafish OI is located in hindbrain rhombomeres 7 and 8 and is organized internally along both the rostro-caudal and dorsal-ventral axes, resulting in a gradient of neuronal persistence times [15]. Neurotransmitter identities as well as axonal projection patterns have been characterized previously [16-18]. In theoretical models of integration mechanisms $[12-14,19,20]$, the existing recruitment order of integrator neurons is crucial: each neuron carries an eye position threshold and once surpassed, the firing rate is linearly related to the eye position in the ON direction [21-23].

In the cat and primate brain, the OI is located in two nuclei, the nucleus prepositus hypoglossi (NPH) and the medial vestibular nucleus (MVN). It contains position coding neurons, which in addition encode saccadic eye velocity to variable extents $[22,23]$. In the goldfish OI (termed Area I in goldfish) position, neurons typically also encode saccadic velocity [21]. 
The velocity storage mechanism is a second shortterm memory system in the oculomotor hindbrain, which is charged by vestibular or optic flow stimulation via vestibular nuclei and the pretectum/accessory optic system. It supports retinal and global image stabilization and maintains the eye velocity for a certain time after cessation of stimulus movement in an after-response. While the monkey NPH has been reported to encode eye/head velocity during vestibular stimulation [22] as well, in goldfish, such head velocity signals are restricted to an anatomical region termed Area II, which is located rostral to the OI $[7,24-26]$. The low-velocity-encoding neurons have not been functionally identified in zebrafish yet (but see anatomical regions in [2, 25]). Zebrafish readily generate slow-phase optokinetic responses, and therefore, velocity-encoding neurons are needed. However, the VSM is still immature in developing larvae: velocity is only stored for very brief periods of time-if at all $[27,28]$.

In summary, the differential eye position and velocity tuning of zebrafish hindbrain neurons is still elusive but crucial for understanding the functional architecture of the OI and other oculomotor nuclei. Here, we employ stimulus protocols designed to measure eye position and eye velocity encoding independently and reveal an anatomical velocity-to-position gradient in rhombomeres 7 and 8 as well as recruitment orders for eye position and eye velocity during the slow phase of the OKR.

In addition to the position/velocity tuning, we characterize the ocular tuning in this study using a separate stimulus protocol. Since vertebrates possess two eyes, the drive for each eye needs to be binocularly coordinated to facilitate stable perception of the whole visual field. This binocular coordination is a readily observable feature in human and zebrafish oculomotor behaviour: most of the time, both eyes move in the same direction with the same amplitude. Historically, two different mechanisms have been suggested: The two eyes could receive conjugate commands to move together as "one can direct a pair of horses with single reins" (Hering's hypothesis). On the other hand, each eye could be controlled independently so that binocular coordination would need to be learned (Helmholtz' hypothesis, [29, 30]). It remains uncertain how binocular coordination is implemented, with the likelihood that a full explanation contains elements of both theories [31, 32]. Here, we employ monocular and binocular stimulation protocols to drive conjugate and monocular eye movements while measuring neuronal activity. We present evidence for a mixed mono-/binocular code in the hindbrain. Within the abducens nucleus, different neurons are recruited preferentially during binocular versus monocular optokinetic responses, which represents a deviation from a strict final common motor pathway.

\section{Results}

Zebrafish hindbrain neurons group into distinct monoand binocular clusters

To localize and functionally characterize hindbrain neurons active during oculomotor behaviour, we stimulated larvae with patterns of moving gratings to elicit optokinetic responses while measuring GCaMP6f calcium signals in individual neurons (Fig. 1a, b).

Zebrafish show a high degree of binocular coordination: most of the time, the eyes are moved in a conjugate fashion with the notable exception of convergence during prey capture and spontaneous monocular saccades ([33], own observations). In order to assess the binocular coordination within the oculomotor system and to identify the location of internuclear neurons (INNs) and other structures, we applied a stimulus protocol (Fig. 2a) geared to decouple both eyes and reduce the gain of the nonstimulated eye to $<0.1$ by showing a moving grating to the stimulated and a stable grating to the non-stimulated eye ([27], Fig. 2a'). This enabled us to classify neurons according to their innervated eye(s) based on their response profile. The stimulus consisted of stimulus phases driving primarily monocular and conjugate eye movements, respectively. The strong decorrelation of left and right eye movements enabled us to classify the monocular or binocular coding of each neuron (Fig. 2). For the characterization of neuronal response types, we calculated the correlation of neural activity traces with each of 52 regressors formed to identify neurons primarily coding for different kinematic parameters (Fig. 2c, see the "Methods" section). These parameters included eye movement direction, ocular selectivity (which identifies the encoded eye muscle(s) when combined with eye movement direction), eye position tuning, and OKR slow-phase eye velocity tuning. We found that eye motion-correlated neurons were virtually always active during clockwise or counterclockwise binocular stimulation (2380 out of 2508 neurons, from 15 larvae with each recording depth sampled 8 -fold). They only differ from each other with regard to the extent of recruitment during monocular eye movements, while neurons exclusively active during monocular eye movements are virtually absent in the hindbrain.

We identified four primary response types in our hindbrain data: two monocular (M) types with activity for either the left or the right eye (LE, RE), which were also active during the binocular stimulus phase (types MLE and MRE, Fig. 2a', Fig. 3a, b, Additional file 1: Figure S1a-b), and two binocular response types. The binocular response types (types BA and BP, Fig. 2b, b' and Fig. 3c, d) were either active during all three (monocular and binocular) stimulus phases ('binocular always', BA, Fig. 2b), or showed a preference towards binocular eye movements ('binocular preferred', BP, Fig. 2b'). 


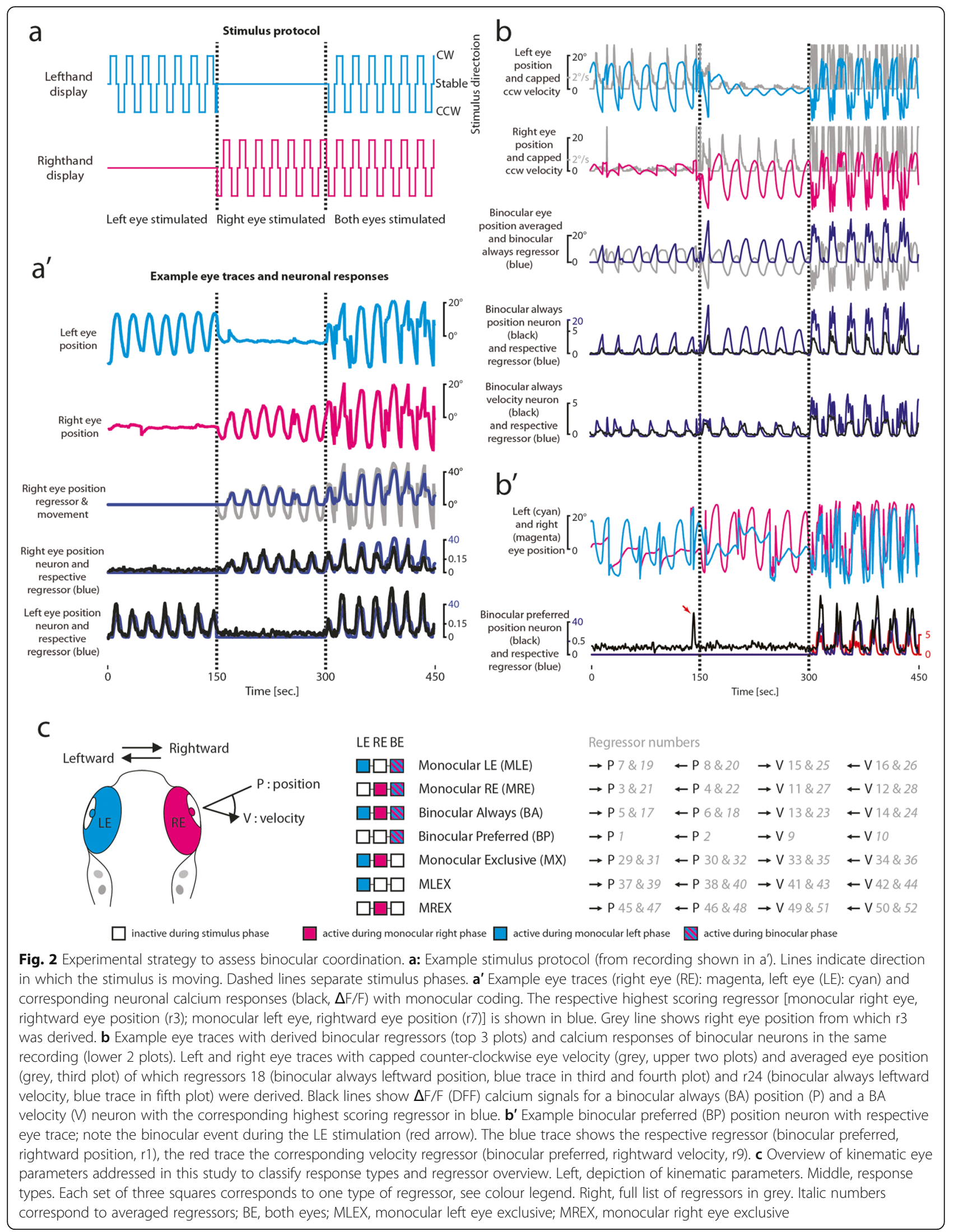




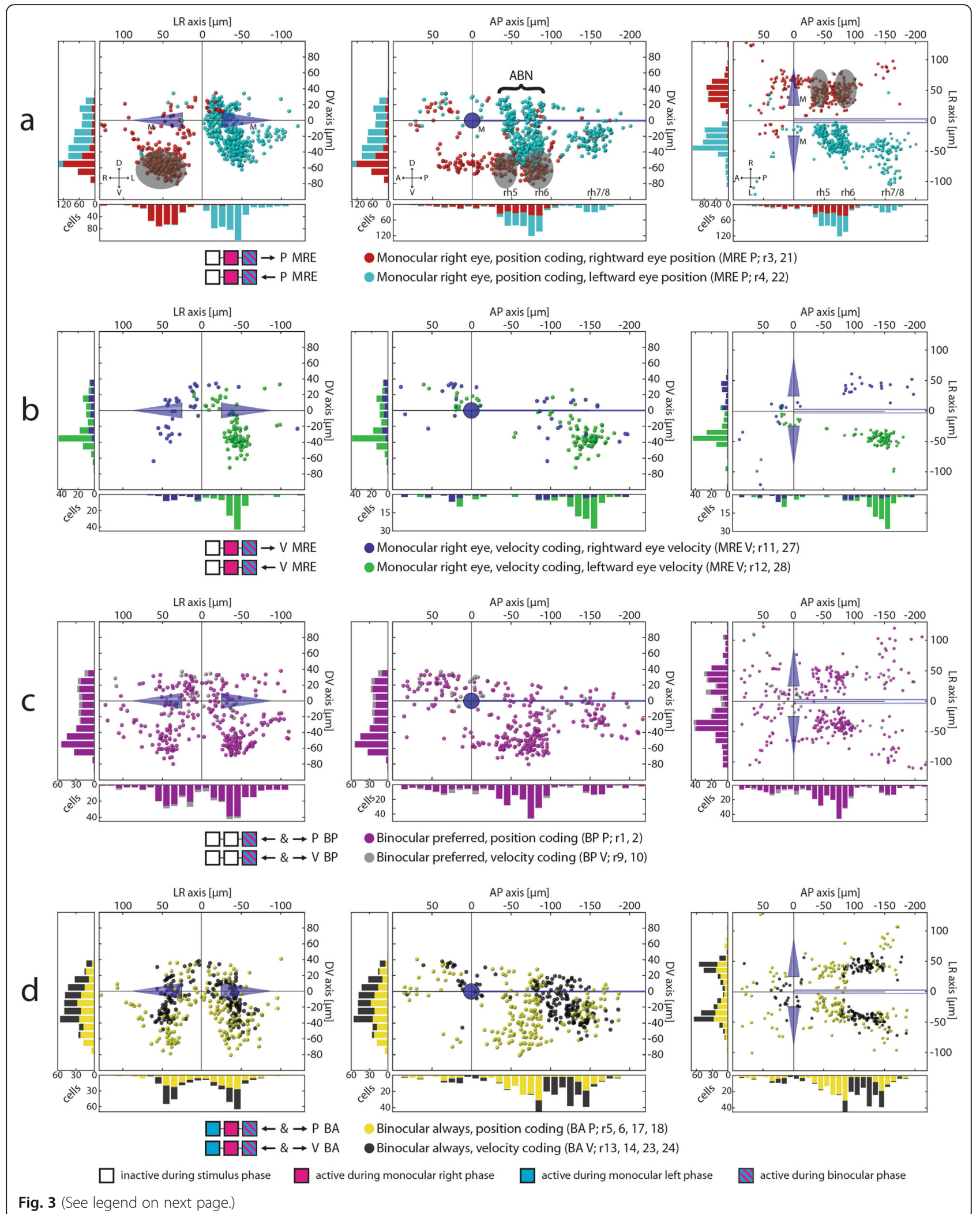


(See figure on previous page.)

Fig. 3 Monocular and binocular cell maps. a-d Transversal, sagittal, and dorsal views for MRE and binocular neurons in the hindbrain (see Additional file 1: Figure S1a-b for mirror-symmetric MLE neurons). Pooled neurons in each figure had the highest correlation to the appropriate regressors mentioned in the legend. A, anterior; $A B N$, abducens nucleus; $B A$, binocular always; $B P$, binocular preferred; $D$, dorsal; $L$, left; $M$, Mauthner cells; MRE, monocular right eye; $\mathrm{P}$, position/posterior; R, right; $r$, regressor; rh 5-8, rhombomeres 5-8; $\mathrm{V}$, ventral/velocity; each coloured ball represents one neuron identified in one fish. Shaded areas in subfigure a show the location of motoneurons expressed in the transgenic Tg(mnx1:TagRFP-T)vu504Tg line (mean vertices position plus standard deviation of three $5 \mathrm{dpf}$ old fish). Sparse, single labelled neurons in the dorsal region have not been highlighted [34]

Since the motor range for eye movements during the binocular stimulation phase was mostly larger than during the monocular phases, we excluded all neurons that did not reach their firing threshold during the monocular phase (Additional file 2: Figure S2, Additional file 3: Figure S3).

Ninety-eight percent of eye movement-correlated neurons, caudal to the Mauthner cells, responded in an ipsiversive manner (2110 vs. 37), though this hemispheric restriction was less prominent rostral to the Mauthner cells $(63 \%, 228$ vs. 133). Eye movement-correlated neurons on the right side of the hindbrain are thus increasingly active during rightward eye positions (of the left and/or right eye) and vice versa.

\section{Monocular neurons}

Monocular position encoding neurons are primarily located in rhombomeres 5 and 6, forming two distinct columns in each rhombomere (Fig. 3a; Additional file 1: Figure S1a). A second cluster can be seen around $150 \mu \mathrm{m}$ caudal to the Mauthner cells and $40 \mu \mathrm{m}$ lateral to the medial longitudinal fasciculus (MLF). This region in rhombomere $7 / 8$ partially overlaps with the areas previously described as the OI in zebrafish [15-17], extending caudal-ventrally into the inferior olive (IO), which we found is mostly monocular encoding. The putative OI region contains a high number of neurons encoding the position of the contralateral eye and only few neurons encoding the position of the ipsilateral eye. Within our imaged brain volume containing rhombomeres 5 and 6, position neurons coding for the ipsilateral eye span only a narrow band 30 to $70 \mu \mathrm{m}$ ventral to the MLF (Fig. 3a left, Additional file 1: Figure S1a). This brain volume corresponds to the anatomical position of the abducens MNs, which we confirmed using a separate mnx1-transgenic line (Tg(mnx:TagRFP-T)vu504Tg, [35]) to label MNs (see overlapping grey shaded areas in Fig. 3a and Additional file 1: Figure S1a). The activity of neurons in this brain volume mostly matched the ipsilateral connections of these motoneurons to the abducting lateral rectus muscle (Fig. 1a, active for the ipsilateral eye during ipsiversive eye movements). Internuclear neurons carrying the information used to innervate the medial rectus should be located on the contralateral side and respond to contraversive positions. Such putative INNs are abundant and located more medially and dorsally than motoneurons, spanning a wider range from $60 \mu \mathrm{m}$ ventral to around $30 \mu \mathrm{m}$ dorsal to the MLF (see Additional file 1: Figure S1a for the labelled anatomical extents of INNs and MNs). These two clusters of putative moto- and INNs in the $\mathrm{ABN}$ are mirror-symmetrical between monocular left and right eye position encoding neurons (Fig. 4a). The cluster containing monocular neurons encoding movement of the contralateral eye forms again two (sub-) clusters in each hemisphere, one located dorsally, the other one located more ventrally. These two putative INN clusters were separated by a faint gap with fewer neurons 10 to $30 \mu \mathrm{m}$ ventral to the MLF rotated roughly by $20^{\circ}$ along the $\mathrm{RC}$-axis (black arrows in Fig. 4a pointing towards the gap).

Monocular slow phase eye velocity neurons are mainly located ventrally to the MLF in $\operatorname{rh} 7 / 8$ and code for the contralateral eye. They are clustered slightly ventrorostrally to the putative OI position neurons with some overlap between both clusters. As is the case for the monocular position neurons, the rh7/8 region also contains only few monocular velocity coding neurons for the ipsilateral eye. Rostral to these identified velocity neurons, some sparse, ungrouped neurons are located in both hemispheres, extending to the caudal end of rh6 (Fig. 3b; Additional file 1: Figure S1b).

Monocular neurons preferentially active during one monocular stimulation phase and silent during binocular movements (monocular exclusive) were heavily underrepresented for both position and velocity (159 of 2508, Additional file 4: Figure S4). Neurons exclusively active during both monocular stimulation phases were virtually absent (Additional file 5: Figure S5d).

\section{Binocular neurons}

We identified binocular neurons that were always active (BA) or were preferentially active during binocular eye movements (binocular preferred, BP). The vast majority of BP neurons encode eye position, not velocity (Fig. 3c). They overlap with monocular position coding neurons in rhombomere $7 / 8$, but their centre of mass is shifted to a more lateral position. The rightward and leftward tuned BP neurons are distributed in the right and left 


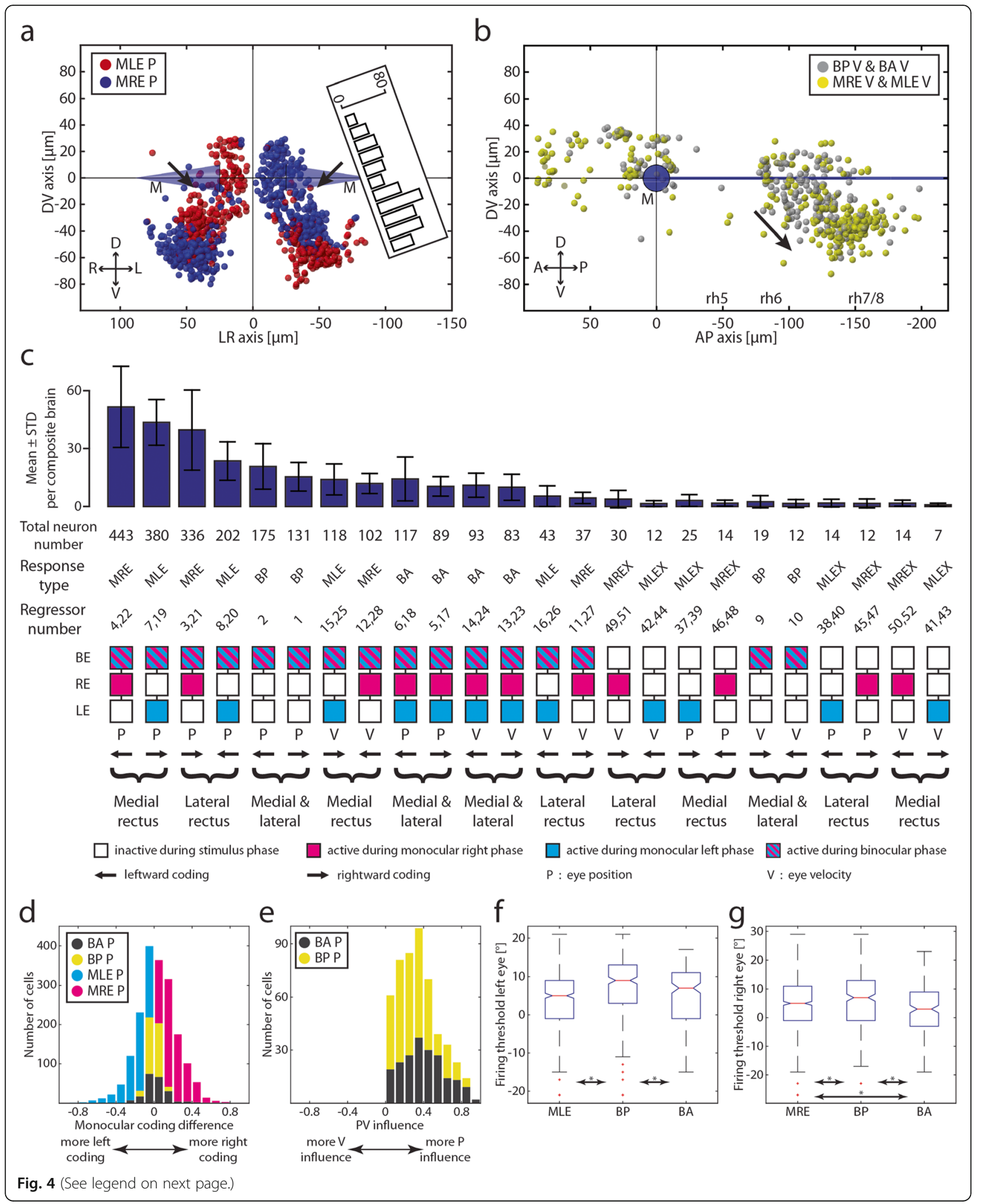


(See figure on previous page.)

Fig. 4 Monocular/binocular synopsis. a Transversal projection of monocular coding neurons within rh5/6 (ABN). D, dorsal; L, left; M, Mauthner cells; MLE, monocular left eye; MRE, monocular right eye; $\mathrm{P}$, position; $\mathrm{R}$, right; $\mathrm{V}$, ventral. Black arrows indicate position of a faint gap between the ventral and dorsal clusters of putative internuclear neurons. Inset shows the numbers of neurons plotted in this figure for the left hemisphere along the $\mathrm{D}-\mathrm{V}$ axis rotated by $20^{\circ}$. b Monocular and binocular velocity encoding neurons. A, anterior; $\mathrm{BA}$, binocular always; $\mathrm{BP}$, binocular preferred; $\mathrm{P}$, posterior; rh 5-8, rhombomere 5-8; V, velocity. Black arrow indicating the direction of the velocity shift. c Sum of the total number of neurons found for each response type sorted pairwise according to the affected muscle(s). The bar plot shows the mean and standard deviation for eight composite brains. BA, binocular always; BP, binocular preferred; MLE, monocular left eye; MLEX, monocular left eye exclusive; MRE, monocular right eye; MREX, monocular right eye exclusive. $\mathbf{d}$ Monocular coding differences for all four main response types for position coding neurons. Index running from -1 (exclusively coding for left eye) to +1 (right eye). e PV influence for BA P and BP P neurons. Index running from -1 (exclusive velocity influence) to +1 (exclusive position influence). $\mathbf{f}, \mathbf{g}$ Left and right eye firing thresholds acquired during the firing threshold analysis pooled in ON direction

hemispheres, respectively, as expected from the ipsiversive coding scheme. In the $\mathrm{ABN}, \mathrm{BP}$ neurons are clustered more ventrally than neurons encoding eye movements monocularly. Furthermore, more BP neurons were found in the left hemisphere than in the right hemisphere (100 vs. 144; caudal to the Mauthner cells). We do not think that this discrepancy necessarily reflects an anatomical asymmetry/lateralization in the zebrafish, but rather was caused by sampling error or history effects from the stimulus presentation.

Binocular BA-type neurons, which are always active regardless of the stimulated eye or stimulus phase, are homogeneously distributed in the $\mathrm{ABN}$ and putative OI (Fig. 3d), following the pattern of their monocular counterpart, and no lateralization across hemispheres was observed. However, those BA neurons that encode velocity form a narrow band (Fig. 3d, black cells in the right panel) spanning from the dorsal end of rh6 (within our imaged region) to the location of monocular velocity coding neurons in rh7/8 and are absent from the remaining $A B N$ and caudal rh7/8 regions.

While BA neurons responded during all stimulus phases, their responses during monocular stimulus phases were typically smaller than those during binocular stimulus phases, which can likely be attributed to the smaller explored motor range during monocular stimulation (for an assessment of response type classification see the "Methods" section, Additional file 1: Figure S1d).

While monocular and binocular position neurons share roughly the same anatomical locations in the zebrafish hindbrain, an anatomical response type gradient exists for velocity neurons caudal to rh6 (Fig. 4b): binocular velocity neurons are located more rostro-dorsally while monocular velocity neurons form a cluster in the ventral part of $\mathrm{rh} 7 / 8$.

Having identified four primary response types, we next sorted all occurring response types according to the number of identified neurons for each response type and grouped them according to the encoded eye direction (CW, CCW), controlled eye muscles (lateral rectus, medial rectus, or both), and kinematic parameter (eye position or
OKR slow-phase velocity). This analysis (Fig. 4c) revealed that position neurons are more frequent in the hindbrain than slow-phase eye velocity neurons (1938 position vs. 570 velocity). We found more monocular neurons coding for the medial rectus than monocular neurons coding for the lateral rectus eye muscle (1043 medial vs. 618 lateral). Also, using our stimulus protocol, we found more neurons coding for the position of the right eye than for the left eye position (779 right vs. 582 left; this might have been caused by a history dependence, as in $90 \%$ of the recordings the left eye was monocularly stimulated before the right eye). For all mono- and binocular response types, we found neurons dorsal to the MLF and rostral to the Mauthner cells which show an intermingled anatomical distribution of ipsiversive and contraversive response types. This cluster corresponds to the caudal end of the previously described "hindbrain oscillator" (also termed ARTR, [3, 5, 6], Fig. 3, Additional file 1: Figure S1).

To reveal the here reported coding properties of neurons in the hindbrain, we made use of response type classification (Figs. 2, 3, and 4a-c). While this approach is useful to get an overview of the anatomical distributions of the different functionally identified neurons, such classification approach is rather ignorant to the possibility that neuronal responses might form a continuum in-between classified response types. We looked into this issue by first checking the per-neuron difference of correlation to the left versus the right eye (see the "Methods" section). As expected, binocular neurons were located in the centre and had a unimodal distribution, while monocular neurons were distributed more towards the sides caused by the left and right coding population [Fig. 4d, Index running from - 1 (more monocular left eye position coding) to 1 (more monocular right eye position coding)]. The results presented in Fig. $4 \mathrm{~d}$, and other publications [36], indicate that the responsivity of neurons is graded. The oculomotor neuron population forms gradients within the parameter space spanned by the regressors used in our response type classification. Thus, our binary analysis-while providing a useful simplification for understanding the oculomotor 
processing repertoire-disregards the existing functional gradients. Since oculomotor neurons can code for many parameters in parallel, the response type classification can in addition be biased by existing correlations. For example, eye movements during the binocular stimulus phase were faster than during the monocular stimulus phases, which could have resulted in some of the BP position neurons being classified as BP (and not BA) due to a weak encoding of eye velocity in these BP neurons. Furthermore during binocular stimulation, more eccentric eye positions were reached than during monocular stimulation. We checked for these issues by comparing the velocity influence of BA $(n=206)$ and BP $(n=306)$ position coding neurons (see the "Methods" section, Fig. 4e). We found that both groups showed similar velocity-position distributions, with BA position neurons having a slightly stronger position component than BP position neurons (twosided Wilcoxon rank-sum test, $p=5.7^{*} 10^{-7}$, Index running from -1 (velocity) to 1 (position)). The firing thresholds (from the firing threshold analysis, Additional file 2: Figure S2, Additional file 3: Figure S3) of BP position neurons were shifted towards the ON direction compared to BA and monocular position neurons, and for the right eye, BA neurons showed significantly earlier thresholds than MRE neurons (Fig. 4f, g). These observed threshold differences likely result from experimental intricacies such as sampling bias. This control analysis shows that the BP position classification was likely slightly affected by velocity components and a larger dynamic range of eye positions during the binocular stimulation phase, and furthermore, some BP neurons were also active during the monocular stimulation phases, albeit at low activity levels preventing their classification as BA or monocular. Taken together, this suggests that BA and BP neurons might not be two distinctively separate groups but that they exist along a continuum, with the extreme cases being $\mathrm{BA}$ and BP.

\section{Differential encoding of velocity and position in individual neurons}

Our first experiment was geared towards identifying monocular versus binocular tuning. We also classified neurons as either mainly position or mainly velocity encoding (Fig. 3) in this experiment, although intermediate "multi-dimensional" responsivity likely occurs as well. $A B N$ neurons should receive slow-phase velocity signals during optokinetic stimulation, e.g., via the pretectum, vestibular nuclei, cerebellum, and the OI (Fig. 1a' $[8,23$, 37-39]) since a muscle force step is needed to overcome the dampened, viscous kinetics of the oculomotor plant $[40,41]$. In order to investigate the differential coding of oculomotor neurons and to visualize the anatomical distribution of position and velocity coding within rhombomeres $7 / 8$, we developed a binocular closed-loop stimulation protocol to disentangle eye position from eye velocity correlations by eliciting different eye velocities at different eye positions (Fig. 5a-a", see the "Methods" section). This allowed us to consistently evoke combinations of eye position and velocity which would only occur sporadically during optokinetic responses to fixed stimulus sequences. At the same time, the stimulus protocol minimized the occurrence of fast phase eye movements (saccades) in order to improve our ability to relate neuronal activity to slow phase behaviour in this correlative experiment, i.e., the experiment was not designed to identify or characterize the burst system responsible for generating saccades [3, 42]. From the whole recording, we constructed twodimensional tuning curves covering the activity for almost all different eye position and slow phase eye velocity combinations within a certain range (eye position: $-15^{\circ}$ to $+15^{\circ}$, eye velocity: -7 to $+7^{\circ} / \mathrm{s}$, Fig. $5 \mathrm{~b}-\mathrm{d}$, Add itional file 6: Figure S6a-c). Using this protocol, we analysed 889 neurons, which exhibited different combinations of eye position and slow-phase eye velocity tuning. To classify the differences in position and velocity coding for each of these neurons, we calculated a Position-Velocity index $\left(\mathrm{PV}_{\text {Index }}\right)$ based on the correlation of the neuronal response to behavioural regressors (see the "Methods" section). This index runs from -1 (pure velocity coding) to +1 (pure position coding). Both neurons tuned exclusively to position (neuron 1) or velocity (neuron 3 ) exist, as well as intermediate cases (neuron 2, Fig. 5b-d). For neurons with an intermingled position and velocity component $\left(-0.5<\mathrm{PV}_{\text {Index }}<0.5\right)$, the preferred direction was almost always the same for position and velocity $(94 \%, 440 / 470)$.

\section{Firing thresholds of position neurons are distributed across a broad range of eye positions while velocity neurons mainly get activated at velocities close to $0 \%$}

To quantify the neuronal recruitment, we used the twodimensional tuning curves and analysed the activation thresholds for position and velocity in the position and velocity planes intersecting with the origin. This procedure results in one-dimensional eye position tuning curves around eye velocities of $0 \% \mathrm{~s}$ (black and red line in Fig. 5b-d middle panel) and eye velocity tuning curves around eye positions of $0^{\circ}$ (right panel) for the same neurons. Since it is difficult to detect the true onset of action potential firing (firing threshold) using our measured calcium indicator fluorescence signals (see the "Methods" section), the identified activation thresholds were likely positioned slightly in the ON direction relative to the true firing threshold in each neuron. For position encoding neurons $\left(\mathrm{PV}_{\text {Index }}>0, n=\right.$ 533 neurons with identified position threshold), we found that the eye position thresholds are distributed across a broad motor range (roughly $-10^{\circ}$ to $+10^{\circ}$, Fig. 5e). Leftward and rightward eye position encoding neurons had 


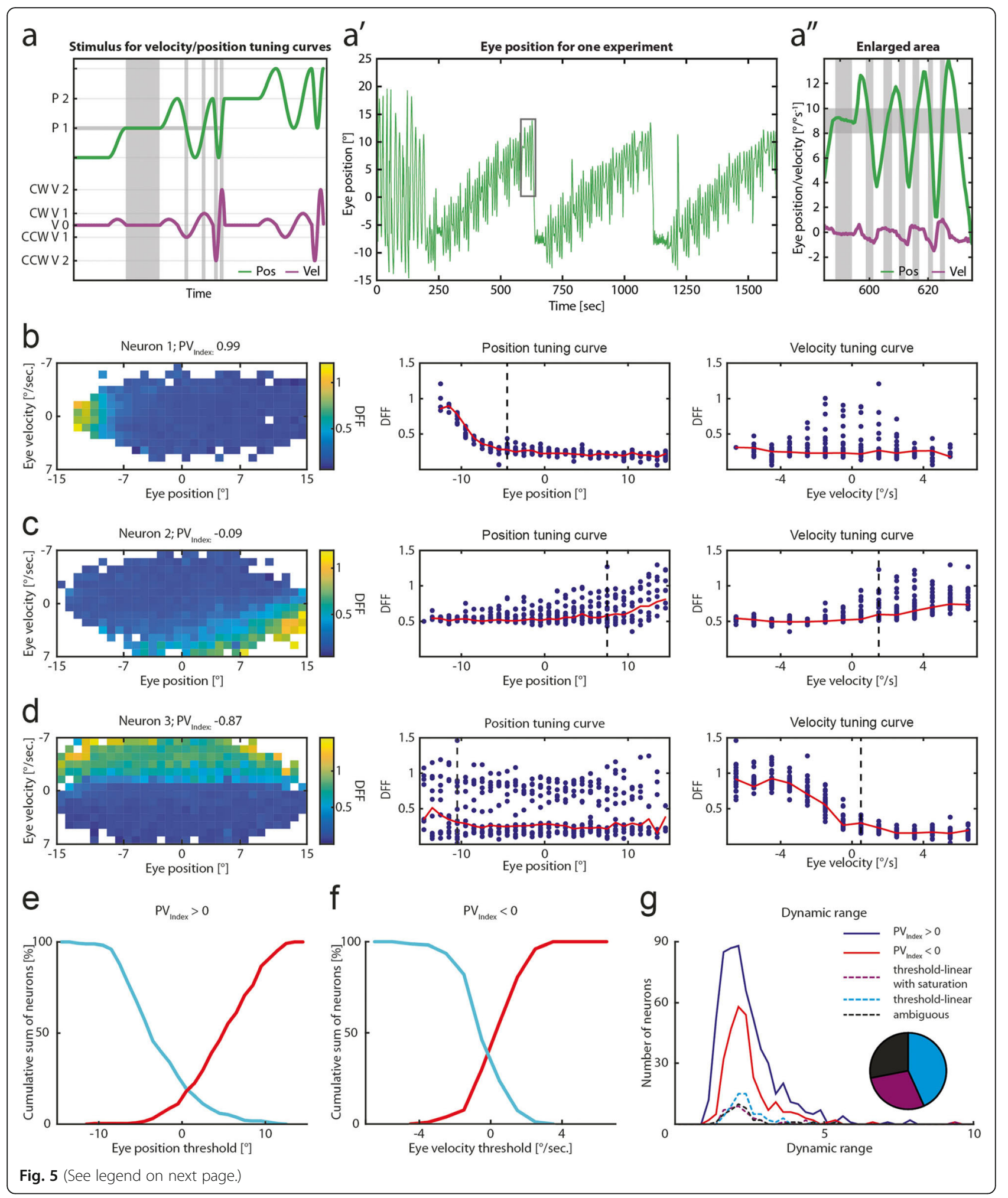


(See figure on previous page.)

Fig. 5 Neuronal tuning for eye velocity and position. a Schematic of the closed loop velocity/position stimulus for highlighted eye position (P1) at different slow-phase eye velocities (CCW V2, CCW V1, V0, CW V1, CW V2). Only two velocity steps are depicted for illustration purposes. Grey shaded rectangles show one eye position bin and different velocities for that bin. CCW, counter-clockwise; CW, clockwise; P, position; V, velocity. a' Example binocular eye trace for one recording. a" Highlighted area from a'. Grey boxes as in a. b-d Left panel, tuning curves showing DFF colour coded for averaged eye position-velocity bins. Middle panel, position tuning curve. Red line shows averaged DFF between $\pm 1 \%$ seye velocity; blue dots for every other eye velocity bin (as in left panel). A black dashed line shows the firing threshold, if identified. Right panel, same as for the middle panel, but for eye velocity. Red line shows averaged DFF between $\pm 1^{\circ}$ eye position. e Cumulative position threshold plot for position coding neurons ( $P V_{\text {Index }}>0$ ) pooled in ON direction to the right (red, $n=250$ ) and left (cyan, $n=283$ ). $\mathbf{f}$ Cumulative velocity threshold plot for velocity coding neurons ( $\left.\mathrm{PV}_{\text {Index }}<0\right)$ pooled in $\mathrm{ON}$ direction to the right (red, $n=104$ ) and left (cyan, $n=175$ ). $\mathbf{g}$ Dynamic range of fluorescence for position and velocity coding neurons ( $P V_{\text {Index }}>0, P V_{\text {Index }}<0$ respectively) and for neuron with a very strong velocity coding ( $P V_{\text {Index }}<-0.5$, dashed lines) separated by their response profile. Pie chart showing the relative numbers for strong velocity coding neurons (w/ saturation, 29\% (40/139); w/o saturation, 43\% (60/139); ambiguous, 28\% (39/139))

slightly different eye position thresholds in our dataset [Wilcoxon rank sum $p=0.000016$, median for rightward coding neurons pooled on ON direction $(n=250): 5.5^{\circ}$, leftward $\left.4.5^{\circ}(n=283)\right]$. Given the small difference, we are not convinced that this discrepancy represents actual asymmetries in the zebrafish larvae, but rather stems from historydependent effects or the optical setup. For the velocityencoding neurons $\left(\mathrm{PV}_{\text {Index }}<0, n=279\right)$, the activation thresholds for velocity mostly span a range between $\pm 2 \%$, so that the calcium signals started to increase at eye velocities close to $0 \% \mathrm{~s}$. Some of the neurons were already active at velocities below $0 \% \mathrm{~s}$ and thus were tuned to both negative and positive velocities. No difference was observed between velocity neurons coding for leftward vs. rightward velocities (Fig. 5f, Wilcoxon rank sum $p=0.24$; rightward $n=104$, leftward $n=175$ ). The strongest fluorescence increases were usually observed after crossing a velocity of $0 \%$ s. However, as mentioned above, the true firing thresholds may start further into the OFF direction $\left(\leq 0^{\circ} / \mathrm{s}\right)$ as (i) we likely could not reliably detect single action potentials using GCaMP6f in our preparation [43] and (ii) our statistical test used to detect thresholds was quite conservative (see the "Methods" section, Additional file 3: Figure S3).

Visual inspection of all strong velocity neurons $\left(\mathrm{PV}_{\text {Index }}<\right.$ $-0.5)$ revealed that some of the identified velocity neurons showed firing saturation at higher velocities (29\%; 40 of 139; Fig. 5g). Calcium indicator saturation, which occurs at high calcium concentrations $\left([\mathrm{Ca}]^{2+}>>\mathrm{K}_{\mathrm{d}}\right.$ ), is unlikely to account for the observed fluorescence saturation, since the dynamic range of fluorescence values $\left(\mathrm{F}_{\mathrm{Max}} / \mathrm{F}_{\mathrm{Min}}\right)$ was (i) much smaller $(\sim 2.5)$ than the published range of the GCaMP6f indicator (51.7) [43] and (ii) similar for non-saturating position neurons and saturating velocity neurons (Fig. $5 \mathrm{~g}$ ).

For the two velocity tuning types (saturating vs. non-saturating), no clear anatomical clustering is visible (Additional file 7: Figure S7) and we therefore merged the corresponding neurons into one group (potentially the non-saturating neurons could still saturate at higher eye velocities not reached in our experimental protocol).
No anatomical gradients of oculomotor tuning thresholds in the hindbrain

In order to investigate topographical arrangements of tuning thresholds in the hindbrain, we generated anatomical maps of firing thresholds for position $\left(P_{\text {Thres }}\right)$ and velocity $\left(V_{\text {Thres }}\right)$ for position neurons with an identified threshold $\left(\mathrm{PV}_{\text {Index }}>0, n=533\right.$, Additional file 8: Figure S8a) and for velocity neurons ( $\mathrm{PV}_{\text {Index }}<0, n=279$, Additional file 8: Figure S8b). Position thresholds do not appear to be anatomically grouped, and no clear anatomical gradient within any of the neuronal clusters could be identified (KruskalWallis test for position threshold differences $p=0.07$; rh5: 214; rh6: 249; rh7/8: 27). We investigated whether MNs (based on anatomical location) are distributed topographically according to position firing threshold, but were unable to identify a significant gradient [Kruskal-Wallis $p=0.22$, Additional file 8: Figure S8a].

Eye velocity thresholds ( $V_{\text {Thres }}$ ) also did not show any spatial clustering, and no gradient could be observed within the hindbrain. No statistical difference was observed (Kruskal-Wallis $p=0.79$; rh5: 11; rh6: 10; rh7/8: 184).

\section{Neurons in rhombomere $7 / 8$ exhibit a velocity-to-position gradient}

The anatomical clusters of position and velocity coding neurons that we identified using the $\mathrm{PV}_{\text {Index }}$ from the closedloop experiment were generally in agreement with those obtained from the separate experiment described above (compare Fig. $6 \mathrm{a}-\mathrm{c}$ to Fig. 3 and Additional file 1: Figure S1). Neurons in the $\mathrm{ABN}$ (rh5/rh6) displayed an average $\mathrm{PV}_{\text {Index }}$ of 0.44 ( \pm 0.23 STD; $n=521)$ indicating position tuning with some minor velocity sensitivity. Within the $A B N$, the velocity component is strongest around a gap (described above in the "Monocular neurons" section, see Fig. 4a, black arrows) inbetween two clusters of neurons $20 \mu \mathrm{m}$ ventral to the MLF. The velocity neurons identified using the velocity-position stimulus reside in the ventral part of rh7/8 and extend into the area caudal to rh6, overlapping with the volumes containing the BA, MLE, and MRE velocity neurons (Fig. 3b-d, Additional file 1: Figure S1b). In the caudal part of 


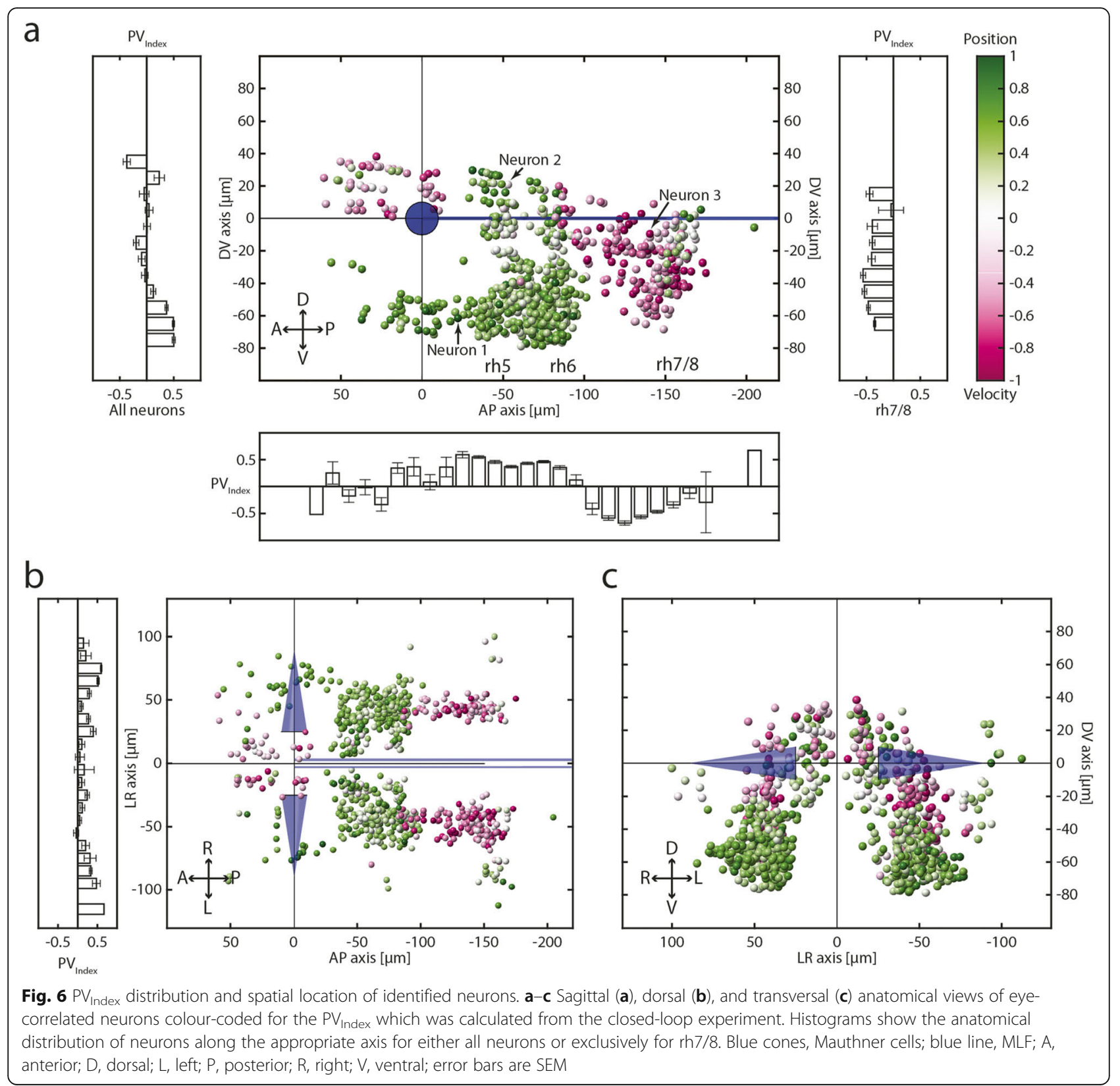

rhombomeres $7 / 8$, we found neurons with more position coding dependence than in the rostral part, especially laterally (Fig. 6a-c). Following the anterior-posterior and ventro-dorsal axes in the caudal hindbrain (rh7/8), our analysis therefore reveals a prominent $\mathrm{PV}_{\text {Index }}$ gradient, shifting from velocity towards an intermingled velocity/position tuning with neurons exhibiting a stronger position coding at the dorso-caudal end.

\section{Discussion}

We investigated the binocular coordination, eye velocity, and position sensitivities, as well as associated recruitment orders and anatomical distributions of oculomotor neurons in the zebrafish hindbrain.

We found four predominant response types, comprised of two monocular and two binocular types (Fig. 7). Monocular neurons consist of MNs, INNs, putative OI, VSM, and IO neurons. We found that abducens INNs are mainly located dorsally to the MNs (Additional file 1: Figure S1a) and together mainly code for eye position (Fig. 7b). In the caudally adjacent rhombomeres 7 and 8, oculomotor neurons mainly code for eye velocity and form a rostro-caudal velocityto-position gradient. No clear segregation between velocity and position encoding neurons could be identified 


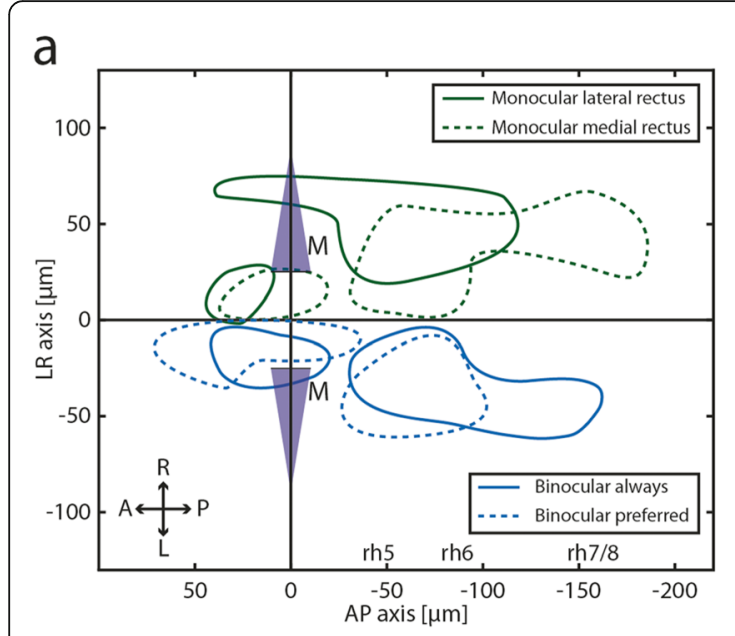

b

\section{C}
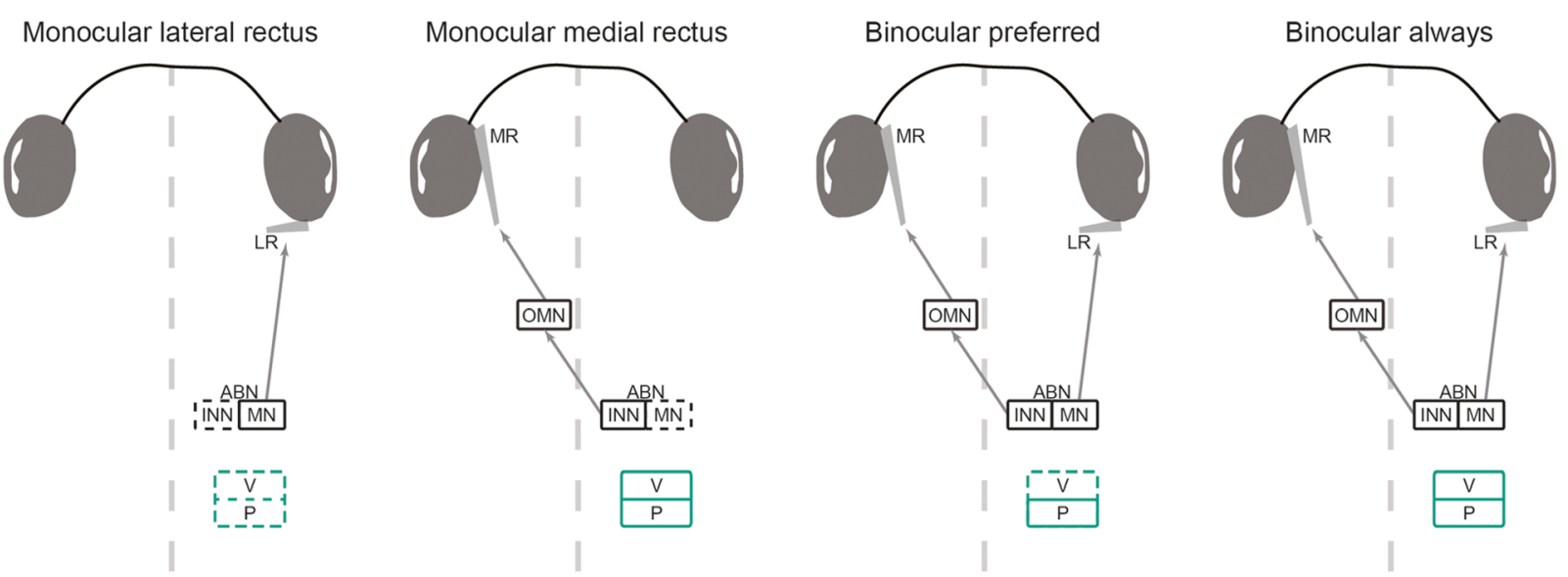

Fig. 7 Summary for binocular coordination and PV encoding in the larval zebrafish hindbrain. a Anatomical separation of monocular and binocular neurons in the dorsal view. For illustrative purposes, all monocular domains are depicted in the right hemisphere, and binocular domains in the left hemisphere (no difference across hemispheres was identified). A, anterior; L, left; M, Mauthner cells; P, posterior; R, right; rh5-8, rhombomere 5-8. b Distinct clusters of eye movement coding neurons in the hindbrain (side view). Arrows indicating position-velocity shift in the OI. D, dorsal; V, ventral. c Schematic illustrating each response type. Note the absence of slow-phase velocity neurons with preferred binocular (BP) encoding and the lack of monocular neurons for the temporal half of the ipsilateral eye outside of the nucleus abducens. Dashed lines represent "missing" neuronal clusters, i.e. only a small numbers of neurons were found for the respective eye movements

in this volume, suggesting that oculomotor integrator and the velocity storage mechanism merge smoothly at this developmental stage. A large fraction of neurons preferentially encode binocular eye movements showing that the recruitment of neurons depends on the executed behaviour (monocular or binocular OKR). Given the number of identified neurons, those coding monocularly for the lateral rectus in OI and VSM are almost absent (Fig. 7c), which is discussed further below.

\section{Anatomical organization of MNs and INNs in the ABN}

To reveal the anatomical volumes containing MNs and INNs in the ABN, we made use of the fact that the lateral rectus eye muscle is innervated by $\mathrm{ABN}$ MNs and should increase its activity during ipsiversive (temporal/ abducting) movements of the ipsilateral eye. We report the location of MNs to be limited to the ventral $A B N$, which is in line with transgenic marker lines for mnx1+ motoneurons (Fig. 3a, Additional file 1: Figure S1a). The INNs are located more dorsally with only a small intermingled zone between the MNs and INNs. This is in line with data from goldfish where ventral $\mathrm{MNs}$ and more dorsal INNs form 4 separate clusters with 2 of them being adjacent and-to some extent-intermingled with each other [44-46].

In our data, we saw a faint gap $(20 \mu \mathrm{m}$ ventral to the Mauthner cells) running along a dorso-lateral to medioventral axis in the cluster of putative INNs, which separates them into two groups (black arrows Fig. 4a). While the dorsal and the ventral domain both carry mainly the 
same information encoding ipsiversive eye positions of the contralateral eye, the dorsal group is in close proximity to a group of neurons recently investigated and identified as the medial vestibular nucleus $(\mathrm{MVN})$ by $\mathrm{D}$. Schoppik and colleagues ([47], which has been registered in the z-brain atlas using the $T g(-6.7 F R h c r t R$ :gal4VP16) line [48]). However, our dorsal group of neurons covered a larger volume and extended more medially than the annotated MVN in the z-brain atlas and mainly coded for eye position, not slow-phase velocity. It is nonetheless possible that the dorsal group partially corresponds to the MVN.

Very ventrally, we found a group of neurons extending rostrally from the pool of rh5 MNs coding for eye position monocularly and binocularly (Fig. 7b, [40 to $40 \mu \mathrm{m}$ on AP axis, $-60 \mu \mathrm{m}$ on DV axis]). As they are not located in the $A B N$ nor labelled in a line specifically labelling MNs (vu504Tg, Fig. 3a), these neurons likely do not project to the extraocular muscles and instead might carry eye movement-related efference copy signals.

\section{Anatomical organization of the caudal hindbrain (rhombomeres 7/8)}

Neurons at the ventro-caudal end of the hindbrain were located very close to the floor plate of the brain, and overlapped with the anatomical location of the inferior olive [49], as were neurons more than $70 \mu \mathrm{m}$ lateral from the MLF in the caudal hindbrain. We did not see a clear anatomical-functional segregation of eye-movementcorrelated putative OI and inferior olive neurons (Additional file 1: Figure S1a). Our results and the previous studies suggest that within our cluster of oculomotor neurons in $\mathrm{rh} 7 / 8$, those located medially $(<60 \mu \mathrm{m}$ from the midline) and rostrally $(-120 \mu \mathrm{m}$ to $-150 \mu \mathrm{m}$ relative to the Mauthner cell), as well as those located medially, caudally $(<-150 \mu \mathrm{m})$, and dorsally (starting $-30 \mu \mathrm{m}$ below the MLF), correspond to the OI (see illustration in Additional file 1: Figure S1), while the ventro-caudal neurons correspond to the inferior olive (compare Fig. $5 g-j$ in [50], Fig. 2 in [16]). Comparing the medio-lateral extent of our putative OI neurons, we did not find neurons closely located to the midline as shown in other studies $[15-17,50]$. As these medially located neurons were reported to be located more dorsally, our recordings might have missed such neurons in dorso-caudal regions. However, in a recent EM study, medially located neurons have been found exclusively at the rostral end of rh7 (boundary to rh6, Fig. 1d and Supplemental Fig. 3 in [17]), an area which we extensively imaged and which contains many velocity-sensitive neurons (rh7) as well as position-sensitive neurons in rh6 (ABN/MVN).

The axonal projection patterns of our reported functional neuron types remain to be identified. The majority of our OI neurons are located ventral to the MLF, likely overlapping with the glutamatergic stripes 1 and 2 [Fig. $2 \mathrm{a}$ in [16]] and the GABAergic stripe S2, which contain both ipsilaterally and contralaterally projecting axons.

\section{Lack of monocular coding for the lateral rectus muscle in the caudal hindbrain}

We show that monocular neurons in rhombomeres $7 / 8$ almost exclusively encode the motion of the contralateral eye in larval zebrafish. In monkeys, it was reported that $50 \%$ of monocular burst-tonic neurons in the nucleus prepositus hypoglossi (NPH) and medial vestibular nucleus (MVN, mammalian equivalents to the OI) code for the ipsi- or contralateral eye during disjunctive fixation/saccades [51], while another study reports "most" (sic) of monocular NPH neurons to be related to the ipsilateral eye [52]. Data from goldfish by Debowy and Baker [53] also shows that only $4 \%$ of neurons in Area I (equivalent to OI) code for the contralateral eye and 57\% for the ipsilateral eye during monocular stimulation.

While Debowy and Baker found almost no monocular integrator neurons coding for the nasal part (medial rectus) of the contralateral eye in goldfish, in the present study, we were missing monocular neurons encoding the temporal hemisphere (lateral rectus) of the ipsilateral eye in zebrafish (Fig. 7c). The rationale for this species difference is unclear and awaits further investigation. In both studies, a lack of monocular coding for one extraocular eye muscle was observed in the oculomotor integrator. The velocity-to-position integration related to this extraocular eye muscle therefore appears to be only encoded in the binocular context.

\section{A mixed, but task-specific monocular-binocular code}

Almost all neurons described in this study were active during conjugate eye movements. According to Hering's hypothesis, monocular eye movements are not effected by monocular signals, but by the summation of binocular signals, which oppose each other in one eye and summate in the other eye, thereby effecting monocular eye movements by means of binocular conjugacy and vergence commands. While we did find BA neurons (whose response profiles are in line with conjugacy commands), the (almost complete) lack of neurons coding for vergence (which would be active only during disconjugate/monocular eye movements in our experiments) is in disagreement with Hering's hypothesis. On the other hand, functional neuron types tuned to a single eye are abundant in the zebrafish hindbrain. These neurons are active regardless of whether the eye movement was monocular or conjugate and their existence conforms to Helmholtz' hypothesis.

The functional structure of the zebrafish ABN shows that recruitment of neuronal pools depends on the 
executed OKR behaviour. The BP pool is preferentially activated during conjugate eye movements and less active during monocular eye movements. The anatomical location of the dominant cluster of $\mathrm{BP}$ neurons in the ventral part of the zebrafish $\mathrm{ABN}$, intermingled with monocular coding neurons and overlapping with the expression of the vu504 line, suggests that many of these BP neurons are indeed MNs. The fact that ABN motoneurons differ in their eye preference and also encode binocular information has previously been shown in monkeys by W. M. King and colleagues ([52, 54-56], discussed in [36]). The functional classification (monocular or binocular encoding) thus does not necessarily correspond to the connected extraocular eye muscle, as ABN motoneurons connect exclusively to the LR muscle of the ipsilateral eye. Our finding represents a deviation from a strict final common pathway: neurons coding for the same eye in different behavioural contexts (binocular vs. monocular OKR) are differentially recruited in these two contexts. Furthermore, if an extraocular motoneuron gets recruited only in certain behavioural contexts (e.g. conjugate eye movements), the lack of motoneuron activity for the innervated eye (e.g. during monocular eye movement) must be compensated by other neurons or elsewhere in the system [57-59] to maintain the eye position. Future studies are needed to reveal how the oculomotor system reconciles this apparent paradox, and the small number of cells involved in the larval zebrafish could facilitate corresponding experiments. Notably, a recent study in Xenopus laevis showed that during the VOR, two distinct motoneuron groups in the abducens can be separated based on their feature code, thus supporting task-specific extraocular motor activity [60].

\section{Recruitment orders for eye position and eye velocity}

The analysis of one-dimensional tuning curves for eye velocity (while keeping eye positions at $0^{\circ}$, Fig. 5) revealed that velocity encoding neurons in the zebrafish hindbrain each increase their firing for one out of the two directions tested, but are not strictly directionselective: a minority of neurons already start firing during slow-phase eye movements into the non-preferred direction. This feature of eye velocity tuning has previously been observed in individual neurons of the goldfish Area II as well (cf. Figure 7b in [26]). However, activations for non-preferred directions were mostly of small magnitude in our data and it remains possible that recording noise or sampling errors affected the identified velocity thresholds. Due to the above-described saturation of velocity signals, a fraction of velocity neurons exclusively encode information for very slow eye velocities, which might enable more precise control of eye velocity in the velocity regime close to $0 \% \mathrm{~s}$. The eye position firing thresholds of position neurons, however, distribute across a broad range of eye positions. This is in agreement with previous reports on the recruitment order in the ABN and OI of other species [21-23, 60-63]. Our analysis of tuning thresholds did not reveal any anatomical gradients for these eye position and velocity thresholds. This includes the MNs located in the ABN (Additional file 8: Figure S8a) for which a soma size gradient has been reported recently [34].

\section{The existing correlations to retinal slip signals remain to be investigated}

In order to generate many and quickly changing eye movements within the limited recording time of our experiments, we chose to use relatively high stimulus velocities. This caused low optokinetic gains [27] and considerable error signals resulting from the remaining retinal slip during slow-phase eye movements. Next to the eye velocity correlations which we describe in this study, these slip signals correlate with the activity of velocity neurons as well. We checked the full dataset of the velocity/position experiment and found that only 4 out of 635 neurons showed a better correlation to a retinal slip signal than to eye position or velocity (correlation analysis, data not shown).

\section{Persistent activity generation likely relies on the observed velocity-to-position gradient in the caudal hindbrain}

Our analysis of differential position versus velocity encoding $\left(\mathrm{PV}_{\text {Index }}\right)$ revealed dominance of position coding in the $\mathrm{ABN}$ (rh 5/6) and an anatomical velocity-to-position gradient of oculomotor neurons in rhombomere $7 / 8$, which have stronger velocity weights in the rostral part of rhombomere $7 / 8$ and stronger position weights in the caudal part.

The rostral part of our identified velocity coding neurons (in rh7) likely corresponds to the velocity storage mechanism (Area II, [7, 24-26]), which is rostrally adjacent to the OI (Area I) in goldfish. While in adult goldfish, a clear functional separation of Areas I and II has been reported, in the larval zebrafish, the velocity and position encoding in rh7/8 appears to form a gradient, making it difficult to draw a border between the velocity storage mechanism and the OI. While the velocity storage mechanism is still maturing in $5 \mathrm{dpf}$ old larval zebrafish (it only stores the velocity for 1 or $2 \mathrm{~s}$ as measured using the optokinetic after-nystagmus ([28], and own observations), the hindbrain already contains a high number of velocity coding neurons.

Our data suggests that the velocity-to-position gradient extends well into the anatomical region of the OI and does not reach exclusive position sensitivity. Therefore, the OI appears to perform only a partial integration (at this developmental stage), where the velocity signals are integrated into an intermediate velocity-position state $[64,65]$. As the semicircular canals were not yet 
functional at the developmental stage used in our experiments [27], future studies using older zebrafish larvae could reveal how vestibular and optokinetic inputs are represented and combined within the OI. The observed gradient is in agreement with a previous publication which identified a change of persistence times in the OI along the rostral-caudal and dorso-ventral axis [15]. These results suggest that integration is achieved by a feed-forward organization of neurons, which gradually change in their position/velocity coding and persistence time. While partial integration can theoretically explain the heterogeneity and spatial gradients of time constants within the integrator, some contradictions to integrator models still remain [66].

It has previously been reported that the activity of the zebrafish OI encodes two separate parameters [67]: while the amplitude of OI neuron activity represents eye position, the spatial pattern of persistent firing represents the context of how the eyes reached that position. If eye positions were reached during optokinetic behaviour, the rostral neurons of the OI showed more persistent activity, while during spontaneous saccadic movement the spatial pattern was reversed. Our results show that in parallel to the previously reported context-dependent anatomical gradient, slow-phase eye velocity is encoded in a similar gradient as well, such that (based on their anatomical rh7/8 location) neurons recruited during OKR are likely to also have a higher velocity sensitivity.

\section{Conclusion}

Our findings characterize the functional layout of the oculomotor hindbrain in zebrafish. They reveal the functional oculomotor architecture regarding a set of key parameters (monocular/binocular encoding, position/velocity encoding, tuning curves/firing thresholds, and anatomy) useful for future investigations into mechanisms underlying persistent activity and sensorimotor transformations. We provide evidence for a mixed but task-specific binocular code and suggest that generation of persistent activity is organized along the rostro-caudal axis in the larval hindbrain.

\section{Methods}

\section{Fish husbandry}

Zebrafish (Danio rerio) expressing GCaMP6f were used in the experiments [Tg(ubi:nls-GCaMP6f)m1300; Additional file 9: Movie S1]. Larvae were raised in a 14/10 h day/night cycle incubator at $29^{\circ} \mathrm{C}$ in E3 solution containing methylene blue. Fish were kept in a TL/N (nacre; [68]) background, imaged larvae were nacre -/.

\section{Transgenesis}

The $\operatorname{Tg}($ ubi:nls-GCaMP6f)m1300 line was created using the Tol2 transposon system [69] and Gateway cloning (Invitrogen, 12537-023, Version D). Briefly, an attB1 primer
(GGGGACAAGTTTGTACAAAAAAGCAGGCTACCATGGCTCCAAAGAAGAAGCGTAAGGTATGGGTT CTCATCATCATCATC) including Kozak [70] and nls [71] sequences was used to amplify GCaMP6f ([43], Addgene plasmid \#40755 pGP-CMV-GCaMP6f); the ubi promoter [3.5 kb, [72], Addgene plasmid \#27320] was inserted into the pENTR5' plasmid. pENTR5' (ubi), pME (nls-GCaMP6f), and pENTR3' (polyA) sequences were then cloned into the pDestTol2pA2 plasmid via an LR clonase reaction (Thermo Fisher Scientific Gateway ${ }^{\oplus}$ LR Clonase ${ }^{-}$Plus enzyme; \#12538120). Twenty-five nanograms per microliter plasmid DNA and $50 \mathrm{ng} / \mu \mathrm{l}$ Tol2 transposase mRNA were coinjected into single-cell stage embryos (nacre +/-). F2 or fish of later generations were used for data acquisition.

\section{Animal preparation and $2 \mathrm{P}$ imaging}

Larvae (5-7 dpf) were screened for nacre-/- and strong GCaMP expression under an epifluorescence microscope (Nikon SMZ25, Tokyo, Japan). They were mounted in a 35-mm petri dish lid in 1.6\% low melting agarose in E3. The agarose surrounding the eyes was removed to ensure unhindered eye movements [73]. During the experiment, the fish were kept in E3 solution devoid of methylene blue.

\section{Microscope setup}

The setup was based on a previously published study [1]. In short, stimuli were presented as vertical gratings (12 roughly equally spaced, red, vertical bars per $360^{\circ}$ ) rotating horizontally around the larvae on a custom-made LED arena (described in the supplemental information under the "Visual Stimulation" section in [1]). Stimulation and eye movement recordings were achieved via a precursory version of ZebEyeTrack [74] running in the LabVIEW environment (National instruments, Austin, USA) and a CMOS camera (DMK 23UV024, The Imaging Source GmbH, Bremen, Germany). Illumination for the detection of the eyes were provided by $850 \mathrm{~nm}$ IR-LEDs (see Fig. 1b). Note that the 700 lp dichroic reflected only a fraction of the IR-light to the sample, which still sufficed to fill out the hole in the IR-LED ring and thus provide back-illumination of the larval eyes for camera detection. Calcium signals were recorded on a hindbrain patch of $\sim 280 \times 280 \mu \mathrm{m}$ at $2 \mathrm{fps}$ on a MOM microscope (Sutter Instruments, Novato, USA; [75]) using C7319 preamplifier (Hamamatsu Photonics K.K., Hamamatsu, Japan) and Sutter's MScan software (Version 2.3.0.1), a 2-photon IR laser (Coherent Chameleon Vision S; $920 \mathrm{~nm}$ excitation wavelength; Coherent Inc., Santa Clara, USA) and a 25x objective (Nikon CFI75, Tokyo, Japan). Stimulus speed was chosen for each fish individually depending on the experiment conducted (see below and Fig. 2a for an example) in order to preferentially generate robust slow phases covering a large dynamic range of eye positions and minimize the occurrence of quick phases (saccades). 


\section{Stimulus protocol for the experiment on monocular versus binocular motor drive}

The stimulus protocol was subdivided into three parts, each lasting for $150 \mathrm{~s}$. In the first two parts, only one eye received a moving stimulus (hereafter referred to as the stimulated eye) while the other eye received a stationary stimulus, and in the third part, both eyes were stimulated. The binocular zone was blocked by black aluminium foil (BKF12, Thorlabs, Newton, USA) the whole time. Stimulus direction changed every $8-10 \mathrm{~s}$ with a stable stimulus for 2-4 s after each direction change. The average stimulus speed during motion phases across animals was $39^{\circ} \%$ $\mathrm{s} \pm 11^{\circ}$ s (STD). Stimulus parameters were chosen for each fish individually to minimize occurrence of saccades. During monocular stimulation, a stationary vertical grating was shown to the OFF eye to minimize yoking. In 137 recordings the left eye was stimulated first, in 15 the right. For illustration and analysis purposes, the latter were reshaped to match the other recordings. The results of this experiment are shown in Figs. 2, 3, 4, and 7 and Additional files 1, 2, 3, 4, and 5: Figure S1-S5.

\section{Stimulus protocol for the experiment on velocity vs. position neuronal tuning}

In the beginning of this stimulus protocol, an alternating OKR stimulus was presented ( 8 s CW, 8 s CCW, 12 repetitions) which was followed by a closed loop protocol in which successful completion of particular eye position/eye velocity combinations was ensured by real-time eye position monitoring. Here, eye position bins were defined, each $2^{\circ}$ wide. In 57 recordings, bins were defined between $\pm 10^{\circ}$, in 3 recordings between $\pm 8^{\circ}$, which corresponded to the well-explored dynamic range of horizontal eye movements. For each eye position bin, the eyes were first driven via the optokinetic response into this bin and then the stimulus velocity was reduced to zero. If the larva kept its gaze centred within that bin for $4 \mathrm{~s}$, the quality criterion was passed, and if the mean eye position moved outside the respective bin boundaries during the $4 \mathrm{~s}$, this part was repeated until it finished successfully. Then, the eye position passed through each bin in CW and CCW directions with different stimulation speed (baseline speed, $1.2 \times$ and $1.4 \times$ of the baseline speed). If a saccade occurred, the current step of the protocol was repeated. The whole closed loop protocol was repeated three times. The average baseline stimulation speed was $31^{\circ} / \mathrm{s} \pm 13^{\circ} / \mathrm{s}$ (STD). Stimulation speed was altered if fish behaviour changed during the experiment. The results of this experiment are shown in Figs. 5, 6, and 7 and Additional files 6, 7, and 8: Figures S6-S8.

\section{Identification of neurons with oculomotor tuning (data analysis)}

All data analysis was done in MATLAB (MathWorks, Natick, USA). Regions of interest (ROIs) were semi- automatically identified as previously published (Correlation Analysis, 3D mapping [1]). This method was altered such that we could apply several regressors at once to a recording, thus enabling us to identify neurons with different coding features at once. For this purpose, each pixel surpassing the $z$-score threshold for any of the regressors was coloured in the anatomical image according to its absolute maximal $z$-score across regressors, resulting in a heat map. This was done to identify eye movement-related pixels; tighter exclusion criteria are applied later in the analysis pipeline depending on the experiment conducted. Regressors used in this study (averaged across both eyes):

- Rectified low eye velocity (capped at $20^{\circ} / \mathrm{s}$, separate regressors for $\mathrm{CW}$ and $\mathrm{CCW}$ directions)

- Rectified high eye velocity (velocities higher than $20^{\circ}$ s in CW and CCW)

- Angular eye position

Since the GCaMP expression was restricted to the nucleus, all drawn ROIs corresponded to somatic signals.

Each recorded optical slice was manually registered in $x$, $y$, and $z$ planes, to a recorded $z$-stack of the same animal. The Mauthner cells and the medial longitudinal fasciculus (MLF) served as landmarks within the $z$-stack in order to combine data from multiple slices and animals into a single reference coordinate system in which the point on the midline between the Mauthner cell somata served as the origin (based on [1]). This approach accounted for differences in the pitch, roll, and yaw of individual fish. It was ignorant about inter-individual hindbrain size variations.

\section{Binocular coordination experiment data analysis}

Data used in this experiment was recorded from 15 larvae (5-7 days post fertilization, dpf). Recordings in which the eye movements surpassed the yoking index were excluded from analysis ( $28 \%$ of original recordings) beforehand (see Additional file 5: Figure S5b) which resulted in an 8-fold coverage of the imaged hindbrain region, ranging from 30 (dorsal) to $60 \mu \mathrm{m}$ (ventral) in 5- $\mu \mathrm{m}$ intervals around the Mauthner cells (rh 4-8; xy position was kept stable for different $z$-levels, 152 recordings total), due to previous reports of the $\mathrm{ABN}$ and OI location [2, 3, 15-17]. The oculomotor neurons of the caudal hindbrain that have been identified in this study were located mostly ventrally to the MLF stretching from the end caudal of rhombomere 6 to the ventro-caudal end of the brain. OI neurons in larval zebrafish have previously been reported ventral to the MLF and extending to the dorsal part as well $[15-17,50]$. One study reported eye position encoding neurons in $\mathrm{rh} 7 / 8$ to be located more dorsal than other studies, but still overlapping the same volume in the brain [2]. It is therefore possible that we missed some more 
dorsally located OI neurons, because the dorsal parts of the hindbrain were not recorded in this study. However, an optogenetic perturbation study found the maximum effect on integrator performance in rostral areas of the OI 50 to $150 \mu \mathrm{m}$ caudal to the Mauthner cells [20], suggesting that the relevant anatomical regions have been well sampled in this study.

To classify the response quality and type of each neuron, we performed a regression analysis. For each ROI, the $\Delta \mathrm{F} / \mathrm{F}$ (DFF) calcium time series was smoothed using a 5-time-point sliding window kernel filter, with the DFF at the time $k$ :

$$
\mathrm{DFF}_{k}=\frac{\mathrm{DFF}_{k-2} * 0.25+\mathrm{DFF}_{k-1} * 0.5+\mathrm{DFF}_{k}+\mathrm{DFF}_{k+1} * 0.5+\mathrm{DFF}_{k+2} * 0.25}{2.5}
$$

Each eye position trace was offset by its respective median to account for individual resting eye position (negative eye position and eye velocity is defined as left or leftward respectively). The DFF trace of each ROI was then correlated with several traces derived from behavioural data (eye position/velocity), which we refer to as "regressors".

We created regressors based on conservative inclusion criteria. Each regressor was (i) either coding for eye velocity or for eye position, (ii) had different combinations of activity during the individual stimulation phases, and (iii) rectified in plus or minus direction. In addition, we tested two (duplicate) types of regressors sets, one in which the monocular phase activity was derived from the eye trace of the respective eye (for monocular regressors), and one in which this monocular phase activity was derived from the average of both eyes during this stimulation phase. The second set was more reliable for BA neuron identification as the motor range in the monocular phases was smaller than the one in binocular phases in most of the recordings. This resulted in a total of 52 regressors (Additional file 5: Figure S5a+d).

The rectified regressors were then convolved with a "calcium impulse response function" (CIRF) [50] to account for the GCaMP dynamics in our experiments $(1.1 \mathrm{~s}$ measured in vivo by observing exponential signal decay of position encoding neurons after a saccade in the null direction). Velocity was capped at $8 \% \mathrm{~s}$ (the regressor was set to $8 \%$ s if the velocity exceeded $8 \%$ s) to eliminate burst sensitivity (saccade generator). Neuronal ROIs with a correlation of at least 0.6 to any of the regressors were then kept for further analysis.

We excluded neurons from recordings in which the nonstimulated eye responded during monocular stimulus phases (Yoking index threshold, Additional file 5: Figure S5b).

To exclude the possibility that some neurons were erroneously classified as monocular/binocular preferred due to eccentric firing thresholds and the fact that the dynamic eye position range differed during monocular and binocular stimulation (usually it was smaller during monocular stimulation), we calculated the firing threshold during the binocular phase and only kept neurons which reached that threshold during the monocular phases. This resulted in the exclusion of 23\% (732 excluded, 2508 revised and confirmed) of neurons in this follow-up analysis (Additional file 2: Figure S2, Additional file 3: Figure S3).

With the exception of regressors for BA neurons ( $\mathrm{r} 5$, $\mathrm{r} 6, \mathrm{r} 17, \mathrm{r} 18$ for position), we did not observe any notable difference in the location or amount of identified neurons for averaged and non-averaged regressors (Additional file 1: Figure S1c-d). This is explainable by the fact that the motor range was smaller during the monocular phases, and thus, the resulting DFF trace is more representative of the averaged eye position trace (Additional file 5: Figure S5c). As the resulting differences were small, we pooled the corresponding regressors (average and non-averaged ones) for further analysis.

\section{Exclusion of recordings with too much yoking:}

For each eye, the velocity was calculated as the difference of eye position at successive time points. The eye velocity was capped at $8 \%$-to prevent artefacts from saccadesand smoothed (Eq. 1). We calculated a "yoking index" (YI) according to the following equation using sums across time series data points from a given recording:

$$
Y I=\frac{\sum a b s\left(\text { Velocity }_{\text {ON }}\right)-\sum a b s\left(\text { Velocity }_{\text {OFF }}\right)}{\sum a b s\left(\text { Velocity }_{\text {ON }}\right)+\sum a b s\left(\text { Velocity }_{\text {OFF }}\right)}
$$

The YI was calculated for each monocular phase and only recordings where both values were bigger than 0.5 were used in the analysis. The "ON" eye was defined as the stimulated eye (Additional file 5: Figure S5b).

\section{Monocular coding differences}

For each major group of position coding neurons, the correlation coefficient of the highest scoring left and right eye monocular regressor was chosen and the difference in monocular coding was calculated in the following way:

$$
\begin{aligned}
& \text { Monocular coding difference } \\
& =\frac{\text { Corr }_{\text {left }}-\text { Corr }_{\text {right }}}{\text { Corr }_{\text {left }}+\text { Corr }_{\text {right }}}
\end{aligned}
$$

\section{PV influence}

For each BA and BP coding neuron, the velocity influence was calculated by choosing the correlation coefficient of the appropriate velocity regressor depending on the highest scoring regressor used to identify this neuron 
(i.e. if the highest scoring regressor was $\mathrm{r} 2$ it would be compared to r10) according to:

$$
\mathrm{PV}_{\text {Influence }}=\frac{\operatorname{Corr}_{\mathrm{pos}}-\text { Corr }_{\mathrm{vel}}}{\operatorname{Corr}_{\mathrm{pos}}+\operatorname{Corr}_{\mathrm{vel}}}
$$

If the appropriate velocity coefficient was negative, it was set to 0 .

\section{Data analysis for experiment on velocity vs. position neuronal tuning}

Data used in this experiment was collected from 8 recorded fish (5-7 dpf) which resulted in a 6-fold coverage of the imaged hindbrain region (same area imaged as for binocular coordination experiment), ranging from 30 to $-60 \mu \mathrm{m}$ around the Mauthner cells in $10-\mu \mathrm{m}$ intervals, to cover the same area as in the previous experiment (60 recordings total). ROIs were selected as previously described and considered for further analysis if their correlation to any of the rectified position or slow velocity regressors (capped at $8 \% \mathrm{~s}$ ) used in the ROI acquisition exceeded 0.4 (different threshold to previous experiment as this step was only to ensure neurons with position and velocity encoding were still included for downstream analysis). The $\mathrm{PV}_{\text {Index }}$ was calculated based on correlation with the respective highest scoring position and velocity regressor during the closedloop-experiment according to the following equation:

$$
\mathrm{PV}_{\text {Index-Corr }}=\frac{\operatorname{Corr}(\text { Position })-\operatorname{Corr}(\text { Velocity })}{\operatorname{Corr}(\text { Position })+\operatorname{Corr}(\text { Velocity })}
$$

Of 889 neurons approved in the previous analysis, 17 had a negative correlation for either both position or velocity regressors and were thus excluded from this $\mathrm{PV}_{\mathrm{In}}$ dex calculation.

For the 2 dimensional tuning curves, all frames from the recording were used (including OKR stimulation). Frames with a higher eye velocity than $10 \%$ and subsequent three frames were excluded to account for artefacts caused by saccades. Fluorescence was grouped in $1^{\circ}$ eye position bins (from $-15^{\circ}$ to $15^{\circ}$ ) with the appropriate velocity $\left(-7^{\circ} / \mathrm{s}\right.$ to $\left.7^{\circ} / \mathrm{s}\right)$ in bins of $1^{\circ} / \mathrm{s}$ width.

\section{Firing threshold assessment}

To extract the fluorescence activation thresholds (which we used as a proxy for the firing threshold), the smoothed (Eq. 1) and deconvolved (CIRF, see above) DFF was plotted against the binned eye position or velocity $\left(2^{\circ}\right.$ increments for position, $1^{\circ} / \mathrm{s}$ for velocity) tuning curve. Starting three bins from the tail (OFF direction), a one-sided, Bonferroni-corrected Wilcoxon rank-sum test was calculated for each bin against all previous bins combined. The firing threshold was defined as the first point with significant difference to the previous (baseline) data points, where at least one of the following two bins was also significant.

To verify that inactivity of a neuron in the first experiment during a monocular stimulation phase is due to its intrinsic coding properties and not due to a lack of appropriate behaviour, the dynamic eye position range for the monocular phases was compared to the firing threshold during the binocular stimulation. If a neuron did not reach its firing threshold in any monocular phase, it was excluded from further analysis (see Additional file 2: Figure S2).

To verify the robustness of the threshold assessment, we created a bootstrap analysis. This test was the same as the test described above; however, we randomly selected the individual data points from each bin with repetition allowed (e.g. if one bin was comprised of 27 individual measurements we randomly picked 27 times out of the available pool). We then ran this test 1000 times for each neuron and calculated the resultant firing threshold each time. Sorting the thresholds for each neuron, we obtained the 95\% confidence interval (25th and 975th rank) of its activation threshold (for either eye position or eye velocity). These results are shown in Additional file 3: Figure S3.

\section{Statistical information}

Statistical testing was performed using MATLAB (2015a). Non-parametric tests were used as our data did not follow a normal distribution. Statistical significance level was $p<0.05$. For the comparison of firing thresholds in the experiment to determine the velocity and position component, a Kruskal-Wallis test was performed to check for significant differences. Other statistical tests conducted are reported in the appropriate sections.

\section{Chemicals and solutions}

See Table 1.

\section{Table 1 Chemicals}

\begin{tabular}{ll}
\hline Chemical & Supplier \\
\hline $\mathrm{NaCl}$ & Applichem, A3597 \\
$\mathrm{KCl}$ & Carl Roth, 6781.1 \\
$\mathrm{CaCl}$ & AppliChem, A1873 \\
$\mathrm{MgSO} 4$ & Merck, 1.05886 .05000 \\
Methylene blue & AppliChem, A4084 \\
Agarose & Biozym, 850080 \\
\hline E3: $\mathrm{NaCl}(5 \mathrm{mM}), \mathrm{KCl}(0.17 \mathrm{mM}), \mathrm{CaCl}(0.33 \mathrm{mM}), \mathrm{MgSO}_{4}(0.33 \mathrm{mM})$ with $0.01 \%$ \\
methylene blue
\end{tabular}




\section{Supplementary information}

Supplementary information accompanies this paper at https://doi.org/10. 1186/s12915-019-0720-y

Additional file 1: Figure S1. Monocular left eye neurons and examples for single regressors. Additional regressor cell maps for Fig. 3. a-d: Transversal, sagittal and dorsal views for MLE and BA neurons in the hindbrain. The MLE maps in $a-b$ correspond to neurons which are mirror-symmetric with respect to the neurons plotted in Fig. 3a-b. The plot in c show the lack of clustering of neurons identified with two similar, but slightly different, regressors (averaged and non-averaged regressors). Plot $d$ shows the difference for BA P neurons due to the different motor range explored during the monocular and binocular stimulation phases (Methods). A: anterior; ABN: nucleus abducens; BA: binocular always; D: dorsal; L: left; INN: internuclear neurons; IO: inferior olive; M: Mauthner cells; MLE: monocular left eye; MN: motoneurons; MVN: medial vestibular nucleus; Ol: oculomotor integrator; P: position/posterior; R: right; r: regressor; rh 5-8: rhombomeres 5-8; V: ventral/velocity; lines show the rough borders between individual neuronal clusters.

Additional file 2: Figure S2. Firing threshold analysis. Tuning curves during the monocular and binocular stimulus phases for one neuron excluded from further analysis (a) and one neuron included in further analysis (b). a: The upper row shows the neural activity (DFF) color coded during the monocular left eye (left plot), right eye (middle plot) and binocular (right plot) stimulus phases for individual eye position bins. Monocular tuning curves (cyan left eye, magenta right eye) were plotted for the respective monocular stimulus phase and the binocular stimulus phase (black). Only bins with at least three individual data points were used. Red dot shows firing threshold. For this neuron, the eye position never explored the eye position threshold during the monocular stimulus phases, it was thus excluded from further analysis. In the lower right the corresponding eye positions and neural activity (DFF) are plotted versus time. b: Tuning curves and eye positions for one threshold approved neuron. Note that for this neuron, the monocular tuning curves covered the eye position threshold (red dot).

Additional file 3: Figure S3. Firing threshold bootstrap analysis. Bootstrap analysis with $95 \%$ confidence intervals for the threshold estimation. a: Neurons with positive (position coding) PV Index ( $n=592 ; 533$ with identified threshold). b: Neurons with negative (velocity coding) PV Index $(n=280 ; 279$ with identified velocity threshold). Firing thresholds were pooled in ON direction. Red line shows the measured threshold for each neuron, the blue and black dots the respective upper and lower $95 \%$ confidence bounds for 1000 repetitions.

Additional file 4: Figure S4. Cell maps for monocular exclusive neurons. a-d: Transversal, sagittal and dorsal views for MLEX and MREX neurons. A: anterior; D: dorsal; L: left; M: Mauthner cells; MLEX: monocular left eye exclusive; MREX: monocular right eye exclusive; $P$ : position/posterior; R: right; r: regressor; rh 5-8: rhombomeres 5-8; V: ventral/velocity.

Additional file 5: Figure S5. Methods for monocular/binocular analysis. Additional regressor examples for the monocular/binocular coordination experiment. a: Example regressors and respective eye traces. L: left; NA: non-averaged (see Methods); P: position; R: right; V: velocity; eye traces same as in Fig. 2a'; b: Example eye traces for yoking index exclusion. Yl: yoking index; c: Example binocular always (BA) neuron and the highest scoring regressor $r 6$ (non-averaged) with the corresponding averaged regressor (r18) and eye traces they are based upon. d: Overview of all approved and excluded neurons for each regressor based on the firing threshold analysis. e: All derived regressors from recording shown in Fig. 2a-a'. LE: left eye; P: position; RE: right eye; $V$ : velocity; r: regressor.

Additional file 6: Figure S6. Additional tuning curves and firing thresholds for different neuron populations. a-c: Additional tuning curve plot same as in Fig. 5. d-d": Cumulative position threshold plots for position coding neurons ( $P V_{\text {Index }}>0$ ) pooled in ON for motoneurons ( $d$, left: 147, right: 127), internuclear neurons ( $d$ ', left: 95 , right: 94 , both based on their anatomical location) and the caudal hindbrain (d", left: 19, right: 8). e-e": Cumulative velocity threshold plots for velocity coding neurons $\left(P V_{\text {Index }}<0\right)$ pooled in ON for motoneurons (e, left: 0 , right: 1), internuclear neurons (e': left: 14, right: 6) and the caudal hindbrain (e": left: 113, right: 71).
Additional file 7: Figure S7. Velocity neurons with different response profiles show no spatial clustering. a-c: Sagittal, transversal and dorsal view of threshold-linear $(n=60)$, threshold-linear with saturation $(n=40)$ and ambiguous ( $n=39$ ) neurons ( $P V_{\text {Index }}<-0.5$ ) color-coded according to their response type. A: anterior; D: dorsal; L: left; M: Mauthner cells P: posterior; R: right; rh5-8: rhombomeres 5-8; V: ventral.

Additional file 8: Figure S8. Position and velocity thresholds. Transversal, sagittal and dorsal views of position and velocity coding neurons color-coded for their thresholds. a: Position thresholds ( $\left.P_{\text {Thres }}\right)$ color-coded for all position coding neurons ( $\left.P V_{\text {Index }}>0\right)$ with an identified firing threshold pooled in ON direction $(n=533)$. Inset shows thresholds for motoneurons based on their anatomical location (no statistical significance was observed: Kruskal-Wallis $p=0.22 ; n=2,41,98,89,43)$ b: Velocity threshold $\left(V_{\text {Thres }}\right)$ color-coded for all velocity coding neurons $\left(P V_{\text {Index }}<0\right)$ with an identified firing threshold pooled in $\mathrm{ON}$ direction $(n=279)$.

Additional file 9: Movie S1. Z-stack for one example Tg(ubi:n/sGCaMP6f)m1300 fish. This movie shows a z-stack of a Tg(ubi:n/sG(aMP6f)m1300 larvae at $5 \mathrm{dpf}$ imaged under the above mentioned setup (except using a $\times 20 / 1.0$ Zeiss objective) resulting in an imaged area of $450.56 \times 450.56 \mu \mathrm{m}$ in $x$ and $y$ with $0.88 \mu \mathrm{m}$ per slice in $z$. The movie is contrast enhanced and imaged with increased laser power (roughly $33 \mathrm{~mW}$ after the objective) to highlight GCaMP6f expression (same fish as in Fig. 1b).

\section{Acknowledgements}

We would like to thank Wolfgang Driever, Emre Aksay, Michael B. Orger, Christian Machens, Claudia Feierstein, Sebastian Reinig, Kun Wang, Konstantin F. Willeke, and all lab members of the Arrenberg lab for helpful discussions about the project, and Rebecca Meier, Maximilian Wandl, Sabine Götter, and Sabrina Fuchs for excellent fish care. We would like to thank Fenja Gawlas and Philipp Rustler for help with generating transgenic zebrafish lines.

\section{Authors' contributions}

$C B$ and $C L$ recorded the data. $C B$ analysed the data. $C B, C L$, and $A B A$ wrote the manuscript. $A B A$ conceived and supervised the project and secured funding. All authors read and approved the final manuscript.

\section{Authors' information}

Not applicable.

\section{Funding}

This work was funded by the Deutsche Forschungsgemeinschaft (DFG) grants EXC307 (CIN - Werner Reichardt Centre for Integrative Neuroscience) and INST 37/967-1 FUGG, and the Juniorprofessor programme grant from the Ministry of Science, Research, and the Arts of the State of BadenWürttemberg (MWK). The funders had no role in the design, data collection, analysis, and interpretation of data and in the writing of the manuscript.

\section{Availability of data and materials}

All data generated or analysed during this study are included in this published article and its supplementary information files. Data and analysis algorithms used in this study have been uploaded to the public repository G-Node GIN and can be accessed via https://doi.org/10.12751/g-node. 6521de [76]. Additional materials (e.g. fish lines) will be made available and requests should be addressed to the corresponding author A.B.A.

\section{Ethics approval and consent to participate}

All animal procedures conformed to the institutional guidelines of the University of Freiburg, University of Tübingen and local governments (Regierungspräsidium Freiburg, Regierungspräsidium Tübingen).

\section{Consent for publication}

Not applicable.

Competing interests

The authors declare that they have no competing interests. 


\section{Author details}

${ }^{1}$ Werner Reichardt Centre for Integrative Neuroscience and Institute for Neurobiology, University of Tübingen, 72076 Tübingen, Germany. ${ }^{2}$ Graduate Training Centre of Neuroscience, University of Tübingen, 72074 Tübingen, Germany.

Received: 6 June 2019 Accepted: 6 November 2019

Published online: 29 December 2019

\section{References}

1. Kubo F, Hablitzel B, Dal Maschio M, Driever W, Baier H, Arrenberg AB. Functional architecture of an optic flow-responsive area that drives horizontal eye movements in zebrafish. Neuron. 2014:81(6):1344-59 Available from: http://www.ncbi.nlm.nih.gov/pubmed/24656253 .

2. Portugues $R$, Feierstein $C E$, Engert $F$, Orger MB. Whole-brain activity maps reveal stereotyped, distributed networks for visuomotor behavior. Neuron. 2014;81(6): 1328-43. Available from: http://www.ncbi.nlm.nih.gov/pubmed/24656252

3. Wolf S, Dubreuil AM, Bertoni T, Böhm UL, Bormuth V, Candelier R, et al. Sensorimotor computation underlying phototaxis in zebrafish. Nat Commun. 2017; 8(1):1-12 Available from: http://www.nature.com/articles/s41467-017-00310-3.

4. Joshua M, Lisberger SG. A tale of two species: neural integration in zebrafish and monkeys. Neuroscience. 2015;296:80-91 Available from: http://www. ncbi.nlm.nih.gov/pubmed/24797331

5. Ahrens MB, Orger MB, Robson DN, Li JM, Keller PJ. Whole-brain functional imaging at cellular resolution using light-sheet microscopy. Nat Methods. 2013;10(5):413-20 Available from: http://www.nature.com/doifinder/10.1038/ nmeth.2434.

6. Dunn TW, Mu Y, Narayan S, Randlett O, Naumann EA, Yang C-T, et al. Brainwide mapping of neural activity controlling zebrafish exploratory locomotion. Elife. 2016 22;5(MARCH2016):1-29. Available from: https:// elifesciences.org/articles/12741

7. Straka H, Beck JC, Pastor AM, Baker R. Morphology and physiology of the cerebellar vestibulolateral lobe pathways linked to oculomotor function in the goldfish. J Neurophysiol. 2006;96(4):1963-80 Available from: https:// www.physiology.org/doi/10.1152/jn.00334.2006.

8. Masseck OA, Hoffmann K-P. Comparative neurobiology of the optokinetic Reflex. Ann N Y Acad Sci. 2009;1 164(1):430-9 Available from: http://www. ncbi.nlm.nih.gov/pubmed/19645943 .

9. Cochran S, Dieringer N, Precht W. Basic optokinetic-ocular reflex pathways in the frog. J Neurosci. 1984;4(1):43-57 Available from: http://www.jneurosci. org/lookup/doi/10.1523/JNEUROSCI.04-01-00043.1984.

10. Robinson DA. Integrating with neurons. Annu Rev Neurosci. 1989;12:33-45 Available from: http://www.ncbi.n/m.nih.gov/pubmed/2648952.

11. Seung HS. How the brain keeps the eyes still. Proc Natl Acad Sci U S A. 1996;93(23):13339-44 Available from: http://www.pubmedcentral.nih.gov/ articlerender.fcgi?artid=24094\&tool=pmcentrez\&rendertype=abstract.

12. Aksay E, Olasagasti I, Mensh BD, Baker R, Goldman MS, Tank DW. Functional dissection of circuitry in a neural integrator. Nat Neurosci. 2007;10(4):494504. Available from: http://www.pubmedcentral.nih.gov/articlerender. fcgi?artid $=2803116 \&$ tool $=$ pmcentrez\&rendertype $=$ abstract .

13. Nikitchenko M, Koulakov A. Neural integrator: a sandpile model. Neural Comput. 2008 ;20(10):2379-417. Available from: http://www.ncbi.nlm.nih. gov/pubmed/18533820.

14. Fisher D, Olasagasti I, Tank DW, Aksay ERF, Goldman MS. A modeling framework for deriving the structural and functional architecture of a shortterm memory microcircuit. Neuron. 2013;79(5):987-1000 Available from: https://doi.org/10.1016/j.neuron.2013.06.041.

15. Miri A, Daie K, Arrenberg AB, Baier H, Aksay E, Tank DW. Spatial gradients and multidimensional dynamics in a neural integrator circuit. Nat Neurosci. 2011;14(9):1150-9 Available from: https://doi.org/10.1038/nn.2888

16. Lee MM, Arrenberg AB, Aksay ERF. A structural and genotypic scaffold underlying temporal integration. J Neurosci 2015;35(20):7903-20. Available from: http://www.jneurosci.org/cgi/doi/10.1523/JNEUROSCI.3045-14.2015

17. Vishwanathan A, Daie K, Ramirez AD, Lichtman JW, Aksay ERF, Seung HS Electron microscopic reconstruction of functionally identified cells in a neural integrator. Curr Biol. 2017;27(14):2137-47 e3.Available from: http:// linkinghub.elsevier.com/retrieve/pii/S0960982217307303.

18. Kinkhabwala A, Riley M, Koyama M, Monen J, Satou C, Kimura Y, et al. A structural and functional ground plan for neurons in the hindbrain of zebrafish. Proc Natl Acad Sci. 2011;108(3):1164-9 Available from: http:// www.ncbi.nlm.nih.gov/pubmed/21199947.
19. Seung HS, Lee DD, Reis BY, Tank DW. Stability of the memory of eye position in a recurrent network of conductance-based model neurons. Neuron. 2000;26(1):259-71 Available from: http://linkinghub.elsevier.com/ retrieve/pii/S0896627300811551.

20. Gonçalves PJ, Arrenberg AB, Hablitzel B, Baier H, Machens CK. Optogenetic perturbations reveal the dynamics of an oculomotor integrator. Front Neural Circuits. 2014;8(February):10. Available from: http://www. pubmedcentral.nih.gov/articlerender.fcgi?artid=3937552\&tool= pmcentrez\&rendertype $=$ abstract

21. Aksay E, Baker R, Seung HS, Tank DW. Anatomy and discharge properties of pre-motor neurons in the goldfish medulla that have eye-position signals during fixations. J Neurophysiol. 2000;84(2):1035-49 Available from: http:// www.ncbi.nlm.nih.gov/pubmed/10938326.

22. McFarland JL, Fuchs AF. Discharge patterns in nucleus prepositus hypoglossi and adjacent medial vestibular nucleus during horizontal eye movement in behaving macaques. J Neurophysiol. 1992;68(1):319-32 Available from: http://www.ncbi.nlm.nih.gov/pubmed/1517825.

23. Delgado-García JM, Vidal PP, Gómez C, Berthoz A. A neurophysiological study of prepositus hypoglossi neurons projecting to oculomotor and preoculomotor nuclei in the alert cat. Neuroscience. 1989;29(2):291-307 Available from: http://linkinghub.elsevier.com/retrieve/pii/0306452289900584.

24. Pastor AM, De la Cruz RR, Baker R. Eye position and eye velocity integrators reside in separate brainstem nuclei. Proc Natl Acad Sci U S A. 1994;91(2): 807-11 Available from: http://www.ncbi.n/m.nih.gov/pubmed/8290604.

25. Ma L-H, Punnamoottil B, Rinkwitz S, Baker R. Mosaic hoxb4a neuronal pleiotropism in zebrafish caudal hindbrain. Soares D, editor. PLoS One. 2009 Jun 17:4(6):e5944. Available from: http://dx.plos.org/10.1371/journal.pone.0005944

26. Beck JC, Rothnie P, Straka H, Wearne SL, Baker R. Precerebellar hindbrain neurons encoding eye velocity during vestibular and optokinetic behavior in the goldfish. J Neurophysiol. 2006;96(3):1370-82 Available from: http:// www.physiology.org/doi/10.1152/jn.00335.2006.

27. Beck JC, Gilland E, Tank DW, Baker R. Quantifying the ontogeny of optokinetic and vestibuloocular behaviors in zebrafish, medaka, and goldfish. J Neurophysiol. 2004;92(6):3546-61 Available from: http://jn. physiology.org/cgi/doi/10.1152/jn.00311.2004.

28. Chen C-C, Bockisch CJ, Bertolini G, Olasagasti I, Neuhauss SCF, Weber KP, et al. Velocity storage mechanism in zebrafish larvae. J Physiol. 2014;592(1): 203-14. Available from: http://www.ncbi.n/m.nih.gov/pubmed/24218543 .

29. Bridgeman B, Stark L. The theory of binocular vision. Bridgeman B, Stark L, editors. The theory of binocular vision. Springer US: Boston; 1977. Available from: http://link.springer.com/10.1007/978-1-4613-4148-2

30. Helmholtz H von. Treatise on Physiological Optics, Volume III. Southall JP, editor. Dover Publications; 2005.

31. Coubard OA. Saccade and vergence eye movements: a review of motor and premotor commands. Eur J Neurosci. 2013;38(10):3384-97 Available from: http://www.ncbi.nlm.nih.gov/pubmed/24103028.

32. Chen C-C, Bockisch CJ, Straumann D, Huang MY-Y. Saccadic and postsaccadic disconjugacy in zebrafish larvae suggests independent eye movement control. Front Syst Neurosci. 2016;80. Available from: http:// journal.frontiersin.org/article/10.3389/fnsys.2016.00080

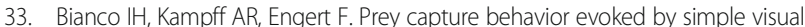
stimuli in larval zebrafish. Front Syst Neurosci. 2011:5(December):1-13 Available from: http://journalffrontiersin.org/article/10.3389/fnsys.2011.00101/abstract.

34. Asakawa K, Kawakami K. Protocadherin-mediated cell repulsion controls the central topography and efferent projections of the abducens nucleus. Cell Rep. 2018;24(6):1562-72 Available from: https://doi.org/10. 1016/j.celrep.2018.07.024.

35. Jao L-E, Appel B, Wente SR. A zebrafish model of lethal congenital contracture syndrome 1 reveals Gle1 function in spinal neural precursor survival and motor axon arborization. Development. 2012;139(7):1316-26 Available from: http://dev.biologists.org/cgi/doi/10.1242/dev.074344.

36. King WM, Zhou W. New ideas about binocular coordination of eye movements: is there a chameleon in the primate family tree? Anat Rec. 2000;261 (4):153-61 Available from: http://doi.wiley.com/10.1002/1097-01 85\%2820000815\%29261\%3A4\%3C153\%3A\%3AAID-AR4\%3E3.0.CO\%3B2-4.

37. Lannou J, Cazin L, Precht W, Le Taillanter M. Responses of prepositus hypoglossi neurons to optokinetic and vestibular stimulations in the rat. Brain Res. 1984;301(1):39-45 Available from: http://linkinghub.elsevier.com/ retrieve/pii/0006899384904001.

38. Leigh RJ, Zee DS. The neurology of eye movements. 4th ed. New York: Oxford University Press; 2006. 
39. Fuchs AF, Kaneko CRS, Scudder CA. Brainstem control of saccadic eye movements. Annu Rev Neurosci. 1985;8(1):307-37 Available from: http:// www.ncbi.n/m.nih.gov/pubmed/3920944.

40. Raphan T, Cohen B. Brainstem mechanisms for rapid and slow eye movements. Annu Rev Physiol. 1978;40(1):527-52 Available from: http:// www.ncbi.nlm.nih.gov/pubmed/205169.

41. Robinson DA. The mechanics of human saccadic eye movement. J Physiol. 1964; 174(2):245-64 Available from: http://doi.wiley.com/10.1113/jphysiol.1964.sp007485.

42. Schoonheim PJ, Arrenberg AB, Del Bene F, Baier H. Optogenetic localization and genetic perturbation of saccade-generating neurons in zebrafish. J Neurosci. 2010;30(20):7111-20. Available from: http://www.pubmedcentral.nih. gov/articlerender.fcgi?artid=3842466\&tool=pmcentrez\&rendertype=abstract .

43. Chen T-W, Wardill TJ, Sun Y, Pulver SR, Renninger SL, Baohan A, et al. Ultrasensitive fluorescent proteins for imaging neuronal activity. Nature. 2013;499(7458):295-300. Available from: http://www.pubmedcentral.nih. gov/articlerender.fcgi?artid=3777791\&tool=pmcentrez\&rendertype=abstract

44. Cabrera B, Torres B, Pásaro R, Pastor AM, Delgado-García JM. A morphological study of abducens nucleus motoneurons and internuclear neurons in the goldfish (Carassius auratus). Brain Res Bull. 1992;28(1):137-44 Available from: http://linkinghub.elsevier.com/retrieve/pii/0361923092902410.

45. Torres B, Pastor AM, Cabrera B, Salas C, Delgado-García JM. Afferents to the oculomotor nucleus in the goldfish (Carassius auratus) as revealed by retrograde labeling with horseradish peroxidase. J Comp Neurol. 1992; 324(3):449-61 Available from: http://doi.wiley.com/10.1002/cne.903240311.

46. Gilland E, Straka H, Wong TW, Baker R, Zottoli SJ. A hindbrain segmental scaffold specifying neuronal location in the adult goldfish, Carassius auratus. J Comp Neurol. 2014:522(10):2446-64 Available from: http//doi.wiley.com/10.1002/cne.23544.

47. Schoppik D, Bianco IH, Prober DA, Douglass AD, Robson DN, Li JMB, et al. Gaze-stabilizing central vestibular neurons project asymmetrically to extraocular motoneuron pools. J Neurosci. 2017;37(47):11353-65 Available from: http://www.jneurosci.org/lookup/doi/10.1523/JNEUROSCI.1711-17.2017.

48. Randlett O, Wee CL, Naumann EA, Nnaemeka O, Schoppik D, Fitzgerald JE, et al. Whole-brain activity mapping onto a zebrafish brain atlas. Nat Methods. 2015;12(11):1039-46 Available from: http://www.nature.com/ doifinder/10.1038/nmeth.3581.

49. Ahrens MB, Li JM, Orger MB, Robson DN, Schier AF, Engert F, et al. Brainwide neuronal dynamics during motor adaptation in zebrafish. Nature. 2012;485(7399):471-7 Available from: http://www.pubmedcentral.nih.gov/ articlerender.fcgi?artid=3618960\&tool=pmcentrez\&rendertype=abstract .

50. Miri A, Daie K, Burdine RD, Aksay E, Tank DW. Regression-based identification of behavior-encoding neurons during large-scale optical imaging of neural activity at cellular resolution. J Neurophysiol. 2011;105(2): 964-80 Available from: http://www.pubmedcentral.nih.gov/articlerender. fcgi?artid=3059183\&tool=pmcentrez\&rendertype=abstract .

51. Sylvestre PA, JTL C, Cullen KE. Discharge dynamics of oculomotor neural integrator neurons during conjugate and disjunctive saccades and fixation. J Neurophysiol. 2003;90(2):739-54 Available from: http://www.physiology. org/doi/10.1152/jn.00123.2003.

52. Zhou W, King WM. Premotor commands encode monocular eye movements. Nature. 1998;393(6686):692-5 Available from: http://www.ncbi. nlm.nih.gov/pubmed/9641680.

53. Debowy O, Baker R. Encoding of eye position in the goldfish horizontal oculomotor neural integrator. J Neurophysiol. 2011;105(2):896-909. Available from: http://www.pubmedcentral.nih.gov/articlerender.fcgi?artid=4103 780\&tool=pmcentrez\&rendertype $=$ abstract

54. King WM, Zhou W, Tomlinson RD, McConville KMV, Page WK, Paige GD, et al. Eye position signals in the abducens and oculomotor nuclei of monkeys during ocular convergence. J Vestib Res. 1994;4(5):401-8. Available from: http://www.ncbi.nlm.nih.gov/pubmed/7994485.

55. Zhou W, King WM. Ocular selectivity of units in oculomotor pathways. Ann N Y Acad Sci. 1996;781(1):724-8 Available from: http://doi.wiley.com/1 0.1111/j.1749-6632.1996.tb15770.x

56. Sylvestre PA, Cullen KE. Dynamics of abducens nucleus neuron discharges during disjunctive saccades. J Neurophysiol. 2002;88(6):3452-68 Available from: http://jn.physiology.org/cgi/doi/10.1152/jn.00331.2002.

57. Miller JM, Davison RC, Gamlin PD. Motor nucleus activity fails to predict extraocular muscle forces in ocular convergence. J Neurophysiol. 2011;105: 2863-73 Available from: http://jn.physiology.org/content/jn/105/6/2863.full.pdf.

58. King WM. Binocular coordination of eye movements - Hering's law of equal innervation or uniocular control? Eur J Neurosci. 2011;33(11):2139-46 Available from: http://doi.wiley.com/10.1111/j.1460-9568.2011.07695.x.
59. Tang X, Büttner-Ennever JA, Mustari MJ, Horn AKE. Internal organization of medial rectus and inferior rectus muscle neurons in the $\mathrm{C}$ group of the oculomotor nucleus in monkey. J Comp Neurol. 2015;523(12):1809-23 Available from: http://doi.wiley.com/10.1002/cne.23760.

60. Dietrich H, Glasauer S, Straka H. Functional organization of vestibulo-ocular responses in abducens motoneurons. J Neurosci. 2017;37(15):4032-45 Available from: http://www.jneurosci.org/lookup/doi/10.1523/ JNEUROSCI.2626-16.2017.

61. Pastor AM, Gonzalez-Forero D. Recruitment order of cat abducens motoneurons and internuclear neurons. J Neurophysiol. 2003;90(4):2240-52 Available from: http://www.ncbi.nlm.nih.gov/pubmed/12801900.

62. Pastor AM, Torres B, Delgado-Garcia JM, Baker R. Discharge characteristics of medial rectus and abducens motoneurons in the goldfish. J Neurophysiol. 1991;66(6):212540 Available from: http://www.physiology.org/doi/10.1152/jn.1991.66.6.2125.

63. Fuchs AF, Scudder CA, Kaneko CR. Discharge patterns and recruitment order of identified motoneurons and internuclear neurons in the monkey abducens nucleus. J Neurophysiol. 1988;60(6):1874-95 Available from: http://www.ncbi.nlm.nih.gov/pubmed/2466962.

64. Anastasio TJ. The fractional-order dynamics of brainstem vestibulooculomotor neurons. Biol Cybern. 1994;72(1):69-79 Available from: http:// link.springer.com/10.1007/BF00206239.

65. Anastasio TJ. Nonuniformity in the linear network model of the oculomotor integrator produces approximately fractional-order dynamics and more realistic neuron behavior. Biol Cybern. 1998;79(5):377-91 Available from: http://link.springer.com/10.1007/s004220050487.

66. Goncalves P. A neural circuit model of the oculomotor integrator: theory for optogenetic dissection. PhD Thesis. 2013;

67. Daie K, Goldman MS, Aksay ERF. Spatial patterns of persistent neural activity vary with the behavioral context of short-term memory. Neuron. 2015;85(4): 847-60 Available from: https://doi.org/10.1016/j.neuron.2015.01.006.

68. Lister JA, Robertson CP, Lepage T, Johnson SL, Raible DW. nacre encodes a zebrafish microphthalmia-related protein that regulates neural-crest-derived pigment cell fate. Development. 1999;126(17):3757-67 Available from: http://www.ncbi.n/m.nih.gov/pubmed/10433906

69. Kwan KM, Fujimoto E, Grabher C, Mangum BD, Hardy ME, Campbell DS, et al. The Tol2kit: a multisite gateway-based construction kit forTol2 transposon transgenesis constructs. Dev Dyn. 2007;236(11):3088-99 Available from: http://doi.wiley.com/10.1002/dvdy.21343.

70. Kozak M. Point mutations close to the AUG initiator codon affect the efficiency of translation of rat preproinsulin in vivo. Nature. 1984;308(5956): 241-6 Available from: http://www.ncbi.nlm.nih.gov/pubmed/6700727.

71. Kalderon D, Roberts BL, Richardson WD, Smith AE. A short amino acid sequence able to specify nuclear location. Cell. 1984;39(3 Pt 2):499-509 Available from: http://www.ncbi.nlm.nih.gov/pubmed/6096007.

72. Mosimann C, Kaufman CK, Li P, Pugach EK, Tamplin OJ, Zon LI. Ubiquitous transgene expression and Cre-based recombination driven by the ubiquitin promoter in zebrafish. Development. 2011;138(1):169-77 Available from: http://dev.biologists.org/cgi/doi/10.1242/dev.059345.

73. Arrenberg AB. Fiber optic-based photostimulation of larval zebrafish. In: Kawakami K, Patton EE, Orger M, editors. Zebrafish: Methods and Protocols. New York, NY: Springer New York; 2016. p. 343-54. (Methods in Molecular Biology; vol. 1451). Available from: https://link.springer.com/content/pdf/1 0.1007\%2F978-1-4939-3771-4.pdf

74. Dehmelt FA, von Daranyi A, Leyden C, Arrenberg AB. Evoking and tracking zebrafish eye movement in multiple larvae with ZebEyeTrack. Nat Protoc. 2018; 13(7):1539-68 Available from: http://www.nature.com/articles/s41596-018-0002-0.

75. Euler T, Hausselt SE, Margolis DJ, Breuninger T, Castell X, Detwiler PB, et al. Eyecup scope_optical recordings of light stimulus-evoked fluorescence signals in the retina. Pflügers Arch - Eur J Physiol. 2009;457(6):1393-414 Available from: http://www.pubmedcentral.nih.gov/articlerender.fcgi?artid= 3037819\&tool=pmcentrez\&rendertype $=$ abstract

76. Brysch C, Leyden C, Arrenberg AB. Data and code for the manuscript "functional architecture underlying binocular coordination of eye position and velocity in the larval zebrafish hindbrain". G-node; 2019. Available from: https://doid.gin.g-node.org/6521de132ffb2638de4b9b57f751b351/

\section{Publisher's Note}

Springer Nature remains neutral with regard to jurisdictional claims in published maps and institutional affiliations. 